AUFSATZ

Martin Mendelski

\title{
Das europäische Evaluierungsdefizit der Rechtsstaatlichkeit
}

\begin{abstract}
"It would not be very difficult to show that the phrase sthe Rule of Law has become meaningless thanks to ideological abuse and general over-use. It may well have become just another one of those self-congratulatory rhetorical devices that grace the public utterances of Anglo-American politicians. No intellectual effort need therefore be wasted on this bit of ruling-class chatter. "
\end{abstract}

\section{Einleitung}

Besitzt die EU die Legitimität und die Fähigkeit, die Rechtsstaatlichkeit ${ }^{2}$ in Mitglieds- und Beitrittsländern zu evaluieren? Ein relativ enger Kreis von Rechtsstaatlichkeits- beziehungsweise Konstitutionalismus-Forschern ${ }^{3}$ beantwortet diese Frage positiv, so wie Michael Blauberger in seinem Artikel im Leviathan, welcher der EU ebenso die Legitimität zu einer solchen Evaluation zuspricht. ${ }^{4}$ Mit diesem Beitrag soll gezeigt werden, dass die bisherige Diskussion im Generellen zu einseitig ist und im Besonderen die Qualität der Rechtsstaatevaluierung durch die EU überschätzt. Die restriktive akademische Debatte beruht auf mehreren fragwürdigen Annahmen, die hiermit auf eine breitere Basis gestellt werden sollen.

Erstens schreiben die meisten Autoren der EU implizit eine demokratie- und rechtsstaatlichkeitsfördernde Wirkung zu und sehen die EU (Europäische Kommis-

1 Shklar 1987, S. 1.

2 Unter Rechtsstaatlichkeit verstehe ich ein multidimensionales sowie historisch, gesellschaftlich und politisch eingebettetes Ordnungsprinzip, das sowohl die formale Qualität des Rechts (formale Legalität), einige Aspekte der substantiellen/materiellen Qualität des Rechts (externe Legalität) als auch die (prozedurale) Qualität der Gesetzgebung und des Justizsystems (Justizkapazität, -unabhängigkeit und -unparteilichkeit, Rechenschaftspflicht) berücksichtigt (siehe im Detail Mendelski 2014; Mendelski 2015). Ich betrachte Rechtsstaatlichkeit nicht als ein umfassendes »thick concept (Tamanaha 2004), sondern schließe die universellen Grundrechte aus (etwa Rede-, Meinungs- oder Versammlungsfreiheit, Recht auf Eigentum). Dies hat einige Vorteile wie zum Beispiel (1) die Vermeidung einer konzeptuellen Uberladung und damit zu starker Überlappung mit verwandten Konzepten (wie zum Beispiel Demokratie und Menschenrechte), (2) mehr Objektivität bei der vergleichenden Analyse, (3) weniger (ideologischer) Missbrauch des Begriffs. Zudem glaube ich, dass Rechtsstaatlichkeit nicht durch Addition der einzelnen Elemente erreicht wird (additiv), sondern durch eine komplementäre Kombination (multiplikativ). Rechtsstaatlichkeit kann somit als Prozess des Balancierens und Kombinierens unterschiedlicher rechtsstaatlicher Prinzipien, Elemente und Dimensionen verstanden werden, welcher von der Einbettung in einer jeweiligen sozialen Ordnung bestimmt wird.

3 Müller 2015; Closa, Kochenov 2016; Jakab, Kochenov 2016; Bogdandy, Sonnevend 2015.

4 Blauberger 2016. 
sion) als legitime Hüterin der Verträge, die das Recht habe, die Rechtsstaatlichkeit zu schützen. Sie bemerken beziehungsweise erwähnen nicht, dass die EU auch negative Wirkungen auf Demokratie und Rechtsstaatlichkeit haben kann, was sich unter anderem im Demokratiedefizit und den "Pathologien der Europäisierung « widerspiegelt. ${ }^{5}$ Zweitens wird kaum berücksichtigt (siehe jedoch Mendelski 2016), dass die EU kein neutraler Akteur ist, sondern ein politischer, der den Rechtsstaatlichkeitsbegriff politisiert und instrumentalisiert. Dies wird zum Beispiel in den politisierten Fortschrittsberichten, der nichtobjektiven, inkonsistenten Evaluierungsmethodik sowie in der einseitigen Rhetorik in der öffentlichen Debatte deutlich. Drittens wird Rechtsstaatlichkeit (obwohl bereits als ein "contested «- und »embedded «-Konzept bestätigt) aus einer begrenzten, konstitutionell-liberalen Perspektive analysiert, was wiederum in einer selektiven und nichtsystematischen Beurteilung der Rechtsstaatlichkeit resultiert.

Mir scheint, dass die meisten der oben genannten Autoren, genauso wie viele liberale Vertreter des Europäischen Parlaments und der Kommission, in die Falle des Gruppendenkens hineingetappt sind, die von einer monistischen Sichtweise der konstitutionellen, liberalen Demokratie - dominiert wird (im Widerspruch zum EU-Leitspruch " united in diversity «). Das einseitige, uniforme Verständnis der liberalen, konstitutionellen Demokratie beziehungsweise Rechtsstaatlichkeit spiegelt sich in der störrischen Befürwortung der Kontrolle und Beschränkung der den demos repräsentierenden parlamentarischen Mehrheit durch sogenannte »unabhängige ", horizontale Rechenschaftsstrukturen ${ }^{6}$ wie zum Beispiel den Verfassungsgerichten, Ombudsmännern, Regulierungsbehörden, Medien etc. wider. Diese einseitige und utopische Sichtweise ist mittlerweile in vielen akademischen und politischen Diskursen zum Grundstandard ("the only game in town ( $^{7}$ ) geworden, neben welcher alternative (das heißt nichtliberale) Perspektiven auf Demokratie (beispielsweise christliche, soziale), Rechtsstaatlichkeit und Rechenschaftsmechanismen keinen Platz haben.

Das Wort "utopisch " benutze ich hier, um auf das defizitäre und pathologische Funktionieren dieser Aufsichts- und Rechenschaftsmechanismen hinzuweisen, die ja durch die EU gefördert, unterstützt und geschützt werden. Dass Rechenschaftsinstitutionen (wie zum Beispiel ein Verfassungsgericht oder eine Anti-Korruptionsbehörde) unter unterschiedlichen kontextuellen Bedingungen unterschiedlich gut beziehungsweise schlecht funktionieren, habe ich in an anderer Stelle ausgeführt. ${ }^{8}$ Dabei fiel insbesondere auf, dass in geschlossenen Gesellschaftsordnungen (»closed access orders ${ }^{9}$ ) horizontale und unabhängige Behörden oft politisiert, gekapert und missbraucht werden, um partikulare Interessen durchzusetzen beziehungsweise zu

5 Börzel, Pamuk 2012; Mendelski 2014; Mendelski 2015; Mendelski 2016.

6 Schedler et al. 1999.

7 Przeworski 1991.

8 Mendelski 2014.

9 North et al. 2009. 
schützen. ${ }^{10}$ Dieser Missbrauch der neu transplantierten oder gestärkten Justizstrukturen, der Justizreformen und der Rechtsstaatlichkeit als "politische Waffe «"11 wird sowohl von EU-feindlichen, »illiberalen « Eliten als auch von EU-freundlichen, "liberalen « Eliten praktiziert. ${ }^{12}$ Tatsächlich agiert die EU in ihrer Bewertung der Rechtsstaatlichkeit nicht neutral und stellt sich (zum Beispiel aus geopolitischen, ideologischen und wirtschaftlichen Gründen) auf die Seite von änderungsfreundlichen »liberalen « Akteuren, obwohl diese dazu tendieren, die Institutionen und Gesetze eines Rechtsstaats ähnlich zu politisieren und zu instrumentalisieren wie "illiberale«, EU-abgeneigte Akteure. ${ }^{13}$

Vor diesem Hintergrund sind die jüngsten »konstitutionellen Krisen « in Rumänien, Ungarn und Polen nicht unbedingt als Rückschritte und Angriffe auf Rechtsstaatlichkeit und Demokratie zu sehen, sondern eher als demokratisch-legitimierte, populistische » Gegenbewegungen «14 gegen die zuvor erlebte Justizialisierung, Politisierung und Instrumentalisierung horizontaler Rechenschaftsstrukturen von oben durch wenige EU-freundliche, änderungsbereite Eliten, die sogenannten "Agenten des Umbaus " ("change agents « ${ }^{15}$ ). ${ }^{16}$ Zudem scheint mir unübersehbar, dass der EU ein entscheidender Beitrag an diesen politisch-konstitutionellen Krisen zukommt, indem sie den Missbrauch und die Politisierung von Justizstrukturen durch EUfreundliche Agenten des Umbaus über Jahre systematisch nicht moniert hat, was wiederum Machtakkumulierung und -missbrauch ermöglichte. In vielen Fällen wurde den änderungswilligen Eliten eine "carte blanche " ausgestellt, die es den "liberalen « Eliten erlaubte, Justizreformen durchzuführen und im gleichen Zug die Rechtsstaatlichkeit zu untergraben. Im Besonderen traten während der Reformen in Osteuropa verschiedene "Pathologien der Europäisierung " auf, ${ }^{17}$ welche die Stabilität, Kohärenz, Generalisierbarkeit und Durchsetzung des Rechts verminderten sowie in erhöhtem Ausmaß die Politisierung der rechtsstaatlichen Strukturen förderte, was wiederum die Rechtsstaatlichkeit und Demokratie beschädigte. ${ }^{18}$

Dieser negativ-pathologische Effekt auf die Qualität des Rechts und den demokratischen Gesetzgebungsprozess ist unter anderem dem Umstand geschuldet, dass

10 Mendelski 2015; Mendelski 2016; siehe auch Ivanova 2013; Capussela 2015; Beširević 2014; Kuzmova 2014; Tudoroiu 2015.

11 Maravall 2003.

12 Natorski 2013; Popova 2012; Di Puppo 2010.

13 Mendelski 2016.

14 Polanyi 1944; Blokker 2016.

15 Magen, Morlino 2009.

16 "Change agents " sind Norm-Entrepreneure, die in "epistemischen Gemeinschaften " (Haas 1992) sozialisiert wurden und als Förderer demokratischer Reformen einen Wandel erreichen wollen. Dazu gehören insbesondere reformbefürwortende Vertreter der heimischen (liberalen) politischen Elite, Bürokratien, Berufsverbände, NGOs, Think Tanks und Akademiker.

17 Börzel, Pamuk 2012; Mendelski 2016.

18 Mendelski 2014; Mendelski 2015; siehe auch Pech 2016; Slapin 2015; Börzel 2015; Börzel 2016; Börzel, Hüllen 2014. 
innerstaatliche, reformbefürwortende Agenten des Umbaus aufgrund der externen Beitrittskonditionalität (dem Zwangsisomorphismus von außen) viele schnelle Anpassungen durch Reformen und beschleunigte, wenig demokratisch Gesetzgebungsverfahren ohne ausführliche Debatten (zum Beispiel Notverordnungen) durchgeführt haben. ${ }^{19}$ Ähnliche Aushöhlungen der Rechtsstaatlichkeit und Demokratie durch EU-Institutionen und ermächtigte technokratische Regierungen wurden mittels Notstandsgesetzgebung und einer "Rhetorik des Notstands « während der Euro-Krise praktiziert. ${ }^{20}$ Die jüngste pessimistische (oder besser: realistische) Evaluierung der EU-Konditionalität durch Europäisierungsforscher reflektiert die empirische Stagnation von Rechtsstaatlichkeitsindikatoren in Südeuropa, ${ }^{21}$ Südosteuropa und früheren post-sowjetischen Staaten sowie der »pathological turn «22 in der Europäisierungsliteratur. ${ }^{23}$

Aufgrund der »pathologischen Macht der EU « ${ }^{24}$ sowie der von ihr geförderten de jure "unabhängigen «, aber de facto politisierten Kontrollinstanzen sollte nicht nur die Frage gestellt werden, wie man nationale Demokratiedefizite verhindert ${ }^{25}$ (zum Beispiel aufgrund des Phänomens der "Tyrannei der Mehrheit «), sondern ebenso, wie man die Tyrannei und den Missbrauch der »unabhängigen « Aufsichtsinstitutionen durch wenige Eliten einschränkt. ${ }^{26}$ Die relevanten Fragen für die Europaforschung und die EU (vor allem in jungen Demokratien, wo Instrumentalisierung und Politisierung der Justiz gang und gäbe sind), müssen demnach lauten: » Who guards the guardians? «27, und im Besonderen: Wer kontrolliert eigentlich die EUfreundlichen politischen und rechtsstaatlichen "change agents «? Die Frage nach der Rechenschaftspflicht der Hüter von nationalen Verfassungen (inklusive von Verfassungsgerichten), der Hüter von Justizunabhängigkeit (Justizräte) und generell von liberalen Agenten des Umbaus wurde bisher kaum gestellt. Sie ist aber hochrelevant, unter anderem weil neuerdings auch Rufe laut werden, dass Verfassungsgerichte und andere »unabhängige « Staatsstrukturen (zum Beispiel Zentralbanken) transnationale hegemoniale Interessen repräsentieren, welche das Gemeininteresse und die durch den demos legitimierten Parlamentsentscheidungen unterminieren. ${ }^{28}$

Die Kerninstitutionen der EU (Kommission, einflussreiche Vertreter des Rates und des Parlaments) fördern diese hegemonialen und (neo)liberalen Interessen sowohl im Bereich der Wirtschaft (neoliberaler und transnationaler Kapitalismus) als auch

19 Sadurski 2004; Risteska 2013; Goetz, Zubek 2007; Mendelski 2015.

20 Kilpatrick 2015; White 2015; Streeck 2015; Maatsch 2015; Kreuder-Sonnen 2016.

21 Matthijs 2014.

22 Mendelski 2016.

23 Siehe zum Beispiel Schimmelfennig, Sedelmeier 2005.

24 Mendelski 2015.

25 Blauberget 2016.

26 Siehe Schmitter 2004.

27 Shapiro 1988.

28 Hirschl 2009; Robinson 2004; Caplan 2005; Hayden 2005. 
in der Politik (liberaler Konstitutionalismus/liberale Demokratie). ${ }^{29}$ Allerdings wird eben nicht berücksichtigt, dass unter bestimmten nicht förderlichen Bedingungen die transplantierten Institutionen (Verfassungsgerichte, Anti-Korruptionsbehörden, Ombudsmann etc.) nicht im Geringsten so unabhängig, demokratisch und rechtsstaatlichkeitsfördernd funktionieren wie in den historisch gewachsenen liberalen Demokratien Westeuropas. Und darin liegt das Problem. Ermächtigte Akteure und neu geschaffene oder gestärkte Institutionen (e.g. Verfassungsgerichte, Anti-Korruptionsbehörden, Regulierungsbehörden, spezielle Gerichte, Zentralbanken) können immer nur so gut funktionieren, wie es ihnen der Kontext (das heißt die soziale Ordnung) erlaubt. Im Besonderen sind innerstaatliche Akteure aus Politik und Justiz in einer sozialen Ordnung eingebettet, die man als geschlossene oder begrenzt zugängliche Ordnung bezeichnen kann, ${ }^{30}$ das heißt eine soziale Ordnung, in der es eingespielte partikularistische Formen des Umgangs gibt, die den westlichen Vorstellungen von Rechtsstaatlichkeit nicht gehorchen. Demnach können rechtsstaatliche Institutionen (Justiz, Verfassungsgerichte etc.) sowohl einen positiv-förderlichen als auch einen negativ-pathologischen Effekt auf Rechtsstaatlichkeit haben, je nachdem, wie die vorhandenen Gesellschafts und-Politikstrukturen auf diese wirken. ${ }^{31}$ Diese entscheidende Differenzierung wird leider von der EU und vielen liberalen Autoren übersehen - und damit die objektive Beurteilung der Rechenschaftsinstitutionen sowie der Rechtsstaatlichkeit eingeschränkt.

Das heißt, so meine These, dass die EU ein Evaluierungsdefizit hat, welches ihr nicht erlaubt, die Entwicklung der Rechtsstaatlichkeit unparteiisch und systematisch zu beurteilen. Das europäische Evaluierungsdefizit zeigt sich insbesondere in drei Problemfeldern:

(1) quantitativer "Je mehr desto besser "-Ansatz zum Nachteil der Qualität,

(2) parteiische, inkonsistente und unsystematische Beurteilung der Rechtsstaat-

lichkeit,

(3) parteiliches »rhetorisches Handeln « in öffentlichen Debatten.

Aufgrund der inadäquaten Methodologie zur objektiven und qualitativen Beurteilung der Rechtsstaatlichkeit ergibt sich, dass, obwohl die Europäische Kommission eine vertragliche Möglichkeit (Artikel 7 EUV) hat, die Grundrechte (zum Beispiel Rechtsstaatlichkeit) der EU de jure zu schützen, ${ }^{32}$ eine $D e$ facto-Legitimität nicht gegeben ist. Aufgrund des Evaluierungsdefizits der EU (welches im Beitrag mithilfe

29 Apeldoorn 2003; Robinson 2004; Blokker 2016.

30 North et al 2009.

31 Mendelski 2014; siehe auch Schwartz 2000.

32 Der Juristische Dienst des Rates verncint eine rechtliche Basis für einen Rahmen zum Schutz der Rechtsstaatlichkeit durch die Kommission und belässt sie allein den Mitgliedsländern. "It is apparent that respect of the values of the Union, including the rule of law, does not as such constitute a Union policy as foreseen by the Treaties. [...] It follows that there is no legal basis in the Treaties empowering the institutions to create a new supervision mechanism of the respect of the rule of law by the Member States, additional to what is laid down in Article 7 TEU, neither to amend, modify or supplement the procedure laid down in this Article. "Siehe http://data.consilium.europa.eu/doc/doc ument/ST-10296-2014-INIT/en/pdf (Zugriff vom 06.07.2016). 
ausgewählter Beispiele zu Verfassungskrisen in Osteuropa dokumentiert wird) ist daher fraglich, ob die EU die Rechtsstaatlichkeit in Zukunft weiterhin beurteilen sollte, solange sie ihre defizitäre Methodik nicht ändert. Allerdings ist eine objektive Methodologie zur (vergleichenden) Evaluierung der Rechtsstaatlichkeit aufgrund der Einbettung des Konzepts in unterschiedlichen historischen, sozialen und politischen Kontexten nur schwer zu bewerkstelligen. Oder anders gesagt, es gibt keine generellen Patentrezepte und »best practices ", wie man Rechtsstaatlichkeit beurteilen, fördern und erreichen kann.

\section{Theorie: Das europäische Evaluierungsdefizit der Rechtsstaatlichkeit}

\subsection{Welches Verständnis hat die EU von Rechtsstaatlichkeit und wie wird diese evaluiert?}

Über viele Jahre hinweg hat die EU die Rechtsstaatlichkeit (beabsichtigt oder unbeabsichtigt) nicht definiert. Ohne eine Konzeptualisierung und Definition der Rechtsstaatlichkeit hatte die Europäische Kommission erheblichen Freiraum, zu entscheiden, ob zum Beispiel ein Beitrittsland die Kopenhagener Kriterien (Beitrittskriterien der EU-Mitgliedschaft) erfüllt oder nicht. Diese vagen Kriterien, über deren Konzepte (Demokratie, Rechtsstaatlichkeit, funktionsfähige Marktwirtschaft) man sich streiten kann, hatten wiederum einigen Ländern (zum Beispiel Bulgarien, Rumänien) trotz schwacher Rechtsstaatlichkeit zu einem »verfrühten « EU-Beitritt verholfen. ${ }^{33}$ Die fehlende Konzeptualisierung der Rechtsstaatlichkeit wurde erst recht spät (im April 2014) von der Europäischen Kommission in einer Mitteilung zum Rechtsstaatsmechanismus nachgeholt. ${ }^{34} \mathrm{Gemäß}$ dieser Mitteilung versteht die Europäische Kommission die Rechtsstaatlichkeit als ein Verfassungsprinzip mit formellen und substantiven Elementen:

"The rule of law is therefore a constitutional principle with both formal and substantive components including several principles: legality, which implies a transparent, accountable, democratic and pluralistic process for enacting laws; legal certainty; prohibition of arbitrariness of the executive powers; independent and impartial courts; effective judicial review including respect for fundamental rights; and equality before the law. «35

Wie man erkennen kann, folgt die EU (sowohl die Kommission, das Parlament als auch der Europäische Gerichtshof) damit einem eher substantiven, werteorientierten »thick «-Konzept der Rechtsstaatlichkeit, bei dem trotz Berücksichtigung prozeduraler Formen der Gesetzgebung relevante Prinzipien der formalen Legalität ("inner morality of law «) - wie zum Beispiel die Stabilität, Kohärenz, Generalisier-

33 Avery 2015.

34 Europäische Kommission 2014. Dabei wurde, wie es scheint, die Definition der Rechtsstaatlichkeit der Venedig-Kommission von 2009 übernommen. Siehe Council of Europe (Venice Commission), Report on the Rule of Law, Study No. 512/2009, CDLAD( 2011)003rev, Strasbourg, 4. April 2011, para. 35.

35 Ebd., S. 3. 
barkeit und Durchsetzung des Rechts - außer Acht gelassen werden. ${ }^{36}$ Dieses NichtBeachten von Prinzipien der Legalität hat unter anderem zu diversen "legalen Pathologien « (Instabilität, Inkohärenz, Instrumentalisierung des Rechts) in EU-Beitrittsländern Osteuropas geführt. ${ }^{37}$

Wie evaluiert die EU Rechtsstaatlichkeit? Die verschiedenen Instrumente der EU zur Evaluierung der Rechtsstaatlichkeit wurden erst kürzlich beschrieben ${ }^{38}$ und sollen hier nur ganz kurz zusammengefasst werden:

(1) Evaluation und Vergleich (Benchmarking): Dabei kommt unter anderem die Methode des Vergleichs mit der sogenannten besten Praxis (Benchmarking) zum Einsatz, was als "finding and implementing best practices « definiert wird. ${ }^{39}$ Die Benchmarking-Methode (ursprünglich entwickelt, um Produkte und Dienstleistungen zu verbessern) basiert auf dem Einsatz von quantitativen Indikatoren, Richtwerten und komplexen Indexmethodologicn, um » beste Praktiken« zu identifizieren und Reformfortschritte zu vergleichen. Die EU setzt zurzeit das Benchmarking-Instrument im Kooperations- und Kontrollverfahren (CVM) Rumäniens und Bulgariens ein, um Fortschritte auf den Gebieten Rechtsstaatlichkeit und Korruption zu messen (und zu verstärken). Zudem wurden jüngst das »European Justice Scoreboard ${ }^{40}$ und der EU-Anti-Korruptionsreport ${ }^{41}$ entwickelt, um die Effizienz von Justizsystemen und Korruptionsprobleme quantitativ vergleichbar zu machen.

(2) Monitoring: Monitoring, die kontinuierliche Berichterstattung und Überwachung von Reformfortschritten und Implementierung von festgelegten Aktionsplänen, beinhaltet sowohl das Sammeln von Informationen zum Zustand des Justizsystems in einem Land (Screening) als auch die Evaluierung dieser Information, zum Beispiel mithilfe von Indikatoren. Die bekanntesten Monitoring-Berichte sind wohl die halbjährlichen Fortschrittsberichte der EU-Osterweiterung sowie die PostErweiterung-CVM-Fortschrittsberichte für Rumänien und Bulgarien. Daneben gibt es weitere Berichte zur Überwachung der Menschenrechte (zum Beispiel European Commission Annual Report on the Charter, European Parliament Annual Report on Fundamental Rights, EU Agency for Fundamental Rights Annual Report) sowie die jährlichen Berichte des europäischen Ombudsmannes über das Funktionieren von EU-Institutionen (European Ombudsman Annual Report). Im Juni 2014 wurde zusätzlich für Mitgliedstaaten "ein neuer EU-Rahmen zur Stärkung des Rechtsstaatsprinzips « eingeführt für Fälle »eines systemischen Zusammenbruchs, der die Integrität, die Stabilität und das ordnungsgemäße Funktionieren der auf nationaler Ebene für die Wahrung der Rechtsstaatlichkeit eingerichteten Organe und Mecha-

36 Siehe Fuller 1969, S. $46 \mathrm{ff}$.

37 Siehe Mendelski 2014; Mendelski 2015; Mendelski 2016.

38 Beispielsweise Carrera et al. 2013.

39 Camp 1995.

40 Siehe http://ec.europa.eu/justice/effective-justice/scoreboard/index_en.htm (Zugriff vom 06.07.2016).

41 Siehe http://ec.europa.eu/dgs/home-affairs/what-we-do/policies/organized-crime-and-hu man-trafficking/corruption/anti-corruption-report/index_en.htm (Zugriff vom 06.07.2016). 
nismen beeinträchtigt «. ${ }^{42}$ Daneben wird ein »Frühwarnmechanismus « installiert, der einen Dialog der Kommission mit dem betroffenen Mitgliedstaat ermöglicht, um "Lösungen “ für das Problem zu erarbeiten.

(3) Prävention und Sanktionierung: Schließlich bietet das Verfahren nach Artikel 7 EUV Möglichkeiten der Prävention bei neindeutige[r] Gefahr einer schwerwiegenden Verletzung der [...] Werte [der Union] " (Artikel 7 Absatz 1 EUV) und ein Sanktionsverfahren für den Fall »eine[r] schwerwiegende[n] und anhaltende[n] Verletzung " der Werte der Union, einschließlich der Rechtsstaatlichkeit (Artikel 7 Absätze 2 und $3 \mathrm{EUV}$ ) ${ }^{4}{ }^{4}$

Bei der Anwendung dieser Mechanismen wird freilich eine defizitäre Methodik angewendet, die sich in der fehlenden Objektivität, Systematik und Inkonsistenz widerspiegelt. Diese Defizite lassen sich an ausgewählten Bcispielen (Verfassungskrisen in Osteuropa) zeigen.

\subsection{Drei Kernproblematiken des EU-Evaluierungsdefzits der Rechtsstaatlichkeit}

\subsubsection{Benchmarking: Quantität vor Qualität}

Das erste Kernproblem des EU-Evaluierungsdefizits der Rechtsstaatlichkeit zeigt sich in einem größtenteils quantitativen Ansatz, der auf quantitativen Benchmarking-Indikatoren und einer "Je mehr desto besser "-Denkweise beruht. Dieser Ansatz nutzt hauptsächlich quantitative Indikatoren zur Beurteilung der Rechtsstaatlichkeit (wie zum Beispiel die Anzahl der angepassten Gesetze an die "besten internationalen Standards", der unterzeichneten Resolutionen, der Verurteilungen, der Verhaftungen, der organisierten Seminare, der Ressourcen). Die Frage ist aber, ob es Sinn macht, Rechtsstaatlichkeit mithilfe eines quantitativ-additiven Ansatzes zu beurteilen. Resultiert zum Beispiel mehr Unabhängigkeit der Richter automatisch in mehr Rechtsstaatlichkeit, falls diese ihre Unabhängigkeit für Korruption missbrauchen? Resultiert mehr Justizkapazität automatisch in mehr Rechtsstaatlichkeit? Deuten mehr Inhaftierungen von Politikern auf eine erhöhte Unabhängigkeit der Justiz hin, mehr adoptierte Gesetze beziehungsweise unterschriebene Resolutionen auf eine Verbesserung der Rechtsstaatlichkeit? Nicht, wenn man ein multiplikatives, systematisches Verständnis hat, bei dem sich die unterschiedlichen Elemente und Dimensionen der Rechtsstaatlichkeit gegenseitig beeinflussen. ${ }^{44}$ Die Gefahr eines zu starken Fokus auf quantitative Resultate (zum Beispiel die Anzahl der Gesetze oder Verhaftungen) besteht darin, dass in vielerlei Hinsicht qualitative Prozesse und Prozeduren unberücksichtigt bleiben (zum Beispiel die Art und Weise, wie diese Gesetze und Verhaftungen zustande gekommen sind). Zudem können die quantitativen Anreize in verschiedenen Pathologien der Europäisierung resultieren, auf welche im empirischen Teil genauer eingegangen wird.

42 Siehe http://europa.eu/rapid/press-release_IP-14-237_de.htm (Zugriff vom 06.07.2016).

43 Siehe http://europa.eu/rapid/press-release_MEMO-16-62_de.htm (Zugriff vom 06.07.2016).

44 Siehe Mendelski 2014. 


\subsubsection{Parteiische und inkonsistente Evaluierung der Rechtsstaatlichkeit in Berichten}

Das zweite Hauptproblem des EU-Evaluierungsdefizits betrifft die parteiische und inkonsistente Evaluierung der Rechtsstaatlichkeit durch die Europäische Kommission in Fortschrittsberichten. ${ }^{45}$ Die Evaluierungsmethodik der Europäischen Kommission ist vage, flexibel und öffnet somit Tür und Tor zu jeweiligen und parteiischen Ad-hoc-Beurteilungen der Rechtsstaatlichkeit, was auch als Doppelmoral kritisiert wird. ${ }^{46}$ Das Hauptproblem ist die Beeinträchtigung der Evaluation durch eine politische Blickrichtung: Die Europäische Kommission (unter dem Einfluss vieler weiterer Akteure) tendiert dazu, die Rechtsstaatlichkeit besser zu bewerten, wenn EU-freundliche, sogenannte »liberale " Regierungen an der Macht sind, und deutlich schlechter, wenn »illiberale «, EU-abgeneigte, beispielsweise national orientierte Regierungen herrschen, obwohl in vielen Fällen die innerstaatlichen Akteure (zum Beispiel national, westlich oder östlich-orientierte Oligarchen) die Gesetze und die Justiz ähnlich (ungenügend) respektieren. Die nichtobjektive Bewertung der Rechtsstaatlichkeit spiegelt sich unter anderem in der Inkonsistenz der Fortschrittsberichte wider. ${ }^{47}$

\subsubsection{Parteiliches »rhetorisches Handeln « in öffentlichen Debatten}

Judith Shklar hat bereits vor einigen Jahrzehnten darauf hingewiesen, dass das Konzept der Rechtsstaatlichkeit zu einem bedeutungslosen »Geschwätz der herrschenden Klasse "verkommen kann, falls es ideologisch und rhetorisch missbraucht wird. ${ }^{48}$ Heutzutage wird dieser Missbrauch im "rhetorischen Handeln « der EURepäsentanten (aber auch vieler Nicht-EU-Politiker) deutlich. "Rhetorisches Handeln " wurde von Frank Schimmelfennig als "strategische Verwendung von normbezogenen Argumenten zur Verfolgung von eigenen Interessen « definiert. ${ }^{49}$ Schimmelfennig nutzt dieses Konzept unter anderem, um die Erweiterung der EU zu erklären. Er zeigte auf, dass Staaten aus Osteuropa ihren Beitritt zur EU mithilfe von Taktiken des » thetorischen Handelns " (Manipulation der europäischen Legitimität, Manipulation der Beitrittskriterien und Aufzeigen von Inkonsistenzen) legitimierten und dadurch ihre Chancen auf die EU-Mitgliedschaft erhöhten. Das Konzept des "rhetorischen Handelns « scheint mir hilfreich, um das EU-Evaluierungsdefizit der Rechtsstaatlichkeit aufzuzeigen, vor allem weil es in der öffentlichen Debatte zur Evaluierung und Messung der Rechtsstaatlichkeit keine eindeutigen Normen, Werte und Indikatoren gibt (sondern nur umstrittene).

In Situationen, bei denen umstrittene Konzepte (wie etwa Rechtsstaatlichkeit) öffentlich (zum Beispiel während der Debatten zu Verfassungskrisen im Europäi-

45 Siehe Kochenov 2008; Schimmelfennig 2012; Carerra et al. 2013; Dimitrov et al. 2014; Toneva-Metodieva 2014.

46 Börzel, Hüllen 2014; Mendelski 2016.

47 Siehe Kochenov 2008.

48 Shklar 1987, S. 1.

49 Schimmelfennig 2001, S. 63. 
schen Parlament) debattiert werden, ist es durchaus möglich, dass Vertreter der EU (Europäische Kommission und Parlamentarier) sowie nationale Vertreter normbezogene Argumente strategisch missbrauchen, um eigene Interessen zu verfolgen. ${ }^{50}$ Als problematisch ist hier zu sehen, dass die EU »rhetorisches Handeln " (ihre Rhetorik des »liberalen Konstitutionalismus «) nicht interessenneutral (zweckneutral) und unparteiisch verwendet, sondern in einer parteilichen Art. Beispielweise wird auf rhetorische Kritik verzichtet, wenn EU-freundliche, änderungswillige Agenten des Umbaus Rechtsstaatlichkeit missachten, was wiederum in einen Vorwurf der Doppelmoral resultiert. Beim parteilichen » rhetorischen Handeln « durch Abgeordnete des Europäischen Parlaments und Vertreter der Europäischen Kommission kommen insbesondere parteiliche Strategien des An-den-Pranger-Stellens (»naming and shaming «) zum Einsatz, die wohl zu kurzfristigen Eingeständnissen seitens der innerstaatlichen Akteure führen, aber nicht zu nachhaltiger Verständigung und Konfliktlösung im Sinne einer offenen, deliberativen Demokratie. ${ }^{51}$ In anderen Worten, die Handlungsmittel, die die EU einsetzt, um ihre Werte zu verteidigen und zu verbreiten, sind "erfolgsorientiert und nicht verständigungsorientiert «. 52

Schließlich wird durch das einseitige »rhetorische Handeln « der EU das umstrittene Konzept der Rechtsstaatlichkeit manipuliert und instrumentalisiert, um eine uniforme, konstitutionell-liberale Vorstellung von ihr zu propagieren und Anhänger von alternativen Vorstellungen über Rechtsstaatlichkeit, Demokratie (und Kapitalismus) zu delegitimieren, was allerdings dem pluralistischen EU-Leitmotiv »united in diversity « widerspricht.

3. Empirie: Evidenz und pathologische Folgen des EU-Evaluierungsdefizits anhand von ausgewählten Beispielen aus Zentral- und Osteuropa

\subsection{Der quantitative Ansatz und seine kontraproduktiven Effekte auf Rechtsstaatlichkeit}

Ein illustratives Beispiel von Evaluierungskriterien eines Twinning-Projekts ${ }^{53}$ aus dem westlichen Balkan dürfte genügen, um den quantitativen Ansatz der EU zu verdeutlichen. Ein typisches Evaluierungsdatenblatt eines Twinning-Projekts zur

50 „Unter diesen Umständen können die Akteure nicht normenreguliert oder angemessen handeln, weil unklar oder umstritten ist, was das der Situation angemessene Handlungsziel oder Handlungsmittel ist. Vielmehr müssen kollektiv geltende Normen erst gesetzt werden, und dazu müssen die beteiligten Akteure entscheiden, welche Werte legitim und richtig sind. Hier kommen die Prozesse des Konflikts über konkurrierende Werte und Normen sowie der Entstehung und Veränderung intersubjektiver handlungsleitender Strukturen ins Spiel. (Schimmelfennig 1997, S. 224)

51 Siehe Habermas 1992.

52 Schimmelfennig 1997, S. 20.

53 Twinning ist eine von der EU zeitlich befristete und finanziell geförderte Verwaltungspartnerschaft zur Unterstützung des Aufbaus von öffentlichen Strukturen im Einklang mit europäischer Verwaltungspraxis in Beitrittskandidatenländern sowie in Ländern ohne Beitrittsperspektive. 
Messung von Fortschritt im Kampf gegen die Korruption und das organisierte Verbrechen nutzt die folgenden quantitativen Indikatoren zur Messung von Reformfortschritt:

»[...] number of serious crime cases dealt with, number of witnesses coming forward, number of meetings, exchange programmes and participants, number of documents produced, number of technical arrangements, number of legislative standards, number of rules of procedure and guidelines drafted and adopted, number of judges and prosecutors trained, number of national key and secondary legislation drafted and adopted, number of legislation and cooperation agreements in place, ratio of information on best practice successfully exchanged and applied, number of training courses provided, number of working groups established. " ${ }^{\mathrm{S}}$

Es ist nicht verwunderlich, dass die von der EU praktizierte "Mehr ist besser «Denkweise zu perversen und pathologischen Effekten führt (beziehungsweise diese verstärkt), die wiederum die Rechtsstaatlichkeit in vielen Beitritts- und Mitgliedsländern untergraben. Die Forderung der EU nach der Übernahme von mehr Gesetzen und der Anpassung an internationale Standards (best practices) führt zwar zu einer Angleichung an westliche Normen (wodurch die externe Legalität gestärkt wird), aber gleichermaßen auch zur Schwächung der »internen Moralität des Rechts " ${ }^{55}$ weil der gesetzliche Rahmen (aufgrund von Reformdruck) oftmals instabiler, inkohärenter und weniger durchsetzbar wird. ${ }^{56}$ Die Forderung der EU nach mehr Justizunabhängigkeit produziert oftmals de jure unabhängigere Justizräte, die dann de facto politisiert sowie intransparenter und weniger rechenschaftspflichtig werden. ${ }^{57}$ Die Forderung der EU nach "soliden Erfolgsbilanzen " im Kampf gegen die Korruption führt zwar zu mehr Verurteilungen, aber gleichermaßen zu mehr Missbrauch der Anti-Korruptionsbehörden und der neu geschaffenen Justizstrukturen sowie zu Verletzungen der Grundrechte. ${ }^{58}$ Die Forderungen der EU nach mehr Justizkapazität zur Erhöhung der quantitativen Effizienz von Justizsystemen (zum Beispiel zur schnelleren Verfahrensbearbeitung) resultieren oft in relativ teuren, auf westlicher Technologie und Software basierenden Informations- und Gerichtsverwaltungssystemen, welche allerdings ohne kontinuierliche Förderhilfen aus dem Ausland (auch in Form von Krediten) kaum nachhaltig sind. ${ }^{59}$ Schließlich ermöglicht die Bereitstellung von vermehrten materiellen Ressourcen (in Form von EUFördergeldern) neue Möglichkeiten für Korruption und leistungslose Dauereinnahmen (»rent seeking «). .60

Insgesamt führt der quantitative Fokus der EU (mit seinen kontraproduktiven Benchmarking-Anreizen) zu pathologischen Wirkungen, die wichtige Kernaspekte der Rechtsstaatlichkeit untergraben. Es scheint auch, dass sich die EU in einem

54 Siehe http://ec.europa.eu/enlargement/pdf/financial_assistance/ipa/2012/multi-beneficia ry/pf5_ipa-2012_winpro-ii_final.pdf (Zugriff vom 06.07.2016).

55 Fuller 1969.

56 Mendelski 2015; Mendelski 2016; siehe Slapin 2015.

57 Mendelski 2015; Bobek, Kosar 2014; Coman 2014.

58 Di Puppo 2010; Mendelski 2012; Mendelski 2016; Ivanova 2013; Capussela 2015.

59 Interview mit einem anonymen USAID-Repräsentanten aus Moldawien.

60 Mungiu-Pippidi 2014. 
"Stabilitäts-Wandel-Dilemma « befindet, ${ }^{61}$ welches zwar innerstaatlichen Wandel durch auferlegte Reformen bewirkt (inklusive der strukturellen Anpassung an westliche Standards), aber gleichzeitig die Gefahr birgt, die »interne Qualität des Rechts « auszuhöhlen und die neugeschaffenen Strukturen politisch zu instrumentalisieren. Dieses (ungelöste) Dilemma trifft vor allem auf viele Ländern Südosteuropas und der ehemaligen Sowjetunion zu, weil es dort aufgrund der erhöhten externen Dependenz und fehlender Überwachungs-Mechanismen leichter ist, die Justiz und Reformen zu instrumentalisieren.

\subsection{Parteiische Evaluierung in Fortschrittsberichten}

Ein aktuelles Beispiel aus Rumänien dürfte die Problematik der parteiischen Evaluierung in Fortschrittsberichten deutlich machen. Rumänien ist, wie die meisten Länder in Südosteuropa, ein Land, das von einer gespaltenen, polarisierten Gesellschaft sowie fragmentierter, instabiler Politik und Politisierung der staatlichen Strukturen geprägt ist. Einerseits gibt es die änderungswilligen, EU-freundlichen liberalen Agenten des Umbaus (PNL-Partei, ehemaliger Präsident Traian Basescu, ehemalige Justizministerin Monica Macovei, liberale, reformbereite Richter und Staatsanwälte). Andererseits gibt es die eher national orientierten sogenannten »illiberalen Veto Player « (die meisten von ihnen Anhänger der PSD-Partei, wie zum Beispiel der ehemalige Ministerpräsident Victor Ponta, sowie deren Anhänger im Justizsystem, Verfassungsgericht etc.). Unter Bedingungen von politisch stark polarisierter Konkurrenz kam es kurz nach dem EU-Beitritt Rumäniens im Jahre 2007 zu politischen Konflikten über den Inhalt von Justiz- und Anti-Korruptionsreformen zwischen Umbaubefürwortern und Umbaugegnern. Diese Machtkonflikte resultierten unter anderem in (gescheiterten und gelungenen) Amtsenthebungsverfahren wichtiger Umbaubefürworter (Basescu, Macovei). Aus objektiver Perspektive kam es in den letzten Jahren auf beiden Seiten zum Missbrauch dieser gesellschaftlichen Veränderungen (Reformen) sowie der Instrumentalisierung der Gesetze und (neu geschaffenen) Justizstrukturen, was wiederum in einem Teufelskreis von Umbau (Reform) und Rückbau (Gegenreform) mündete.

Interessanterweise hat die EU (trotz ihrer Rhetorik von Neutralität und NichtEinmischung) die liberalen, umbaubefürwortenden Agenten durch eine parteiische Evaluierung der Rechtsstaatlichkeit unterstützt. Dies zeigte sich zum Beispiel an der Kritik von Rechenschaftsinstitutionen (Verfassungsgericht, Justizrat), als diese unter der Kontrolle der änderungsunwilligen PSD-Regierung waren. So warf die EU (und ebenso verschiedene liberale Politikberatungseinrichtungen) den PSD-nahen Mitgliedern des rumänischen Justizrats im Jahre 2006 Interessenkonflikte und fehlende Rechenschaftspflicht vor ${ }^{62}$ und schwieg zu ähnlich problematischen Vorfällen (Politisierung, Unregelmäßigkeiten bei der Wahl des CSM-Präsidenten), als der Jus-

61 Mendelski 2016.

62 Europäische Kommission 2006, S. 7. 
tizrat ein paar Jahre später von Reformbefürwortern dominiert wurde. ${ }^{63}$ Die Europäische Kommission erlaubte es sich sogar, mehrere Entscheidungen des rumänischen Verfassungsgerichts zu Anti-Korruptionsreformen zu kritisieren, als das Gericht von PSD-Richtern dominiert wurde, ${ }^{64}$ aber unterstützte das Gericht ein paar Jahre später während der sogenannten Verfassungskrise vom Juli 2012, also auf dem Höhepunkt dieser kumulierten politischen Machtkonflikte zwischen Umbauagenten und »Vetospielern « 65

Insbesondere hat die EU (Europäische Kommission) mit der Veröffentlichung eines kritischen, fehlerhaften und parteiischen CVM-Fortschrittsberichts vom Juli $2012^{66}$ in einen internen politischen Machtkonflikt eingegriffen, mit dem Vorwand, die Rechtsstaatlichkeit zu schützen, die angeblich von der Regierung Ponta zuvor in einer Serie von willentlichen, kurz aufeinanderfolgenden Aktionen untergraben worden war. ${ }^{67}$ Unabhängig davon, ob dieses externe Eingreifen der EU möglicherweise aus einem Dilemma herausführte, so war es doch eindeutig parteiisch und schadete damit zwangsläufig der nachhaltigen Herausbildung von Rechtsstaatlichkeit.

Im CVM-Fortschrittsbericht vom Juli 2012 wurde unter anderem der Missbrauch von "Notstandsverordnungen " durch die Regierung Ponta kritisiert, welche dazu dienten, die Macht der neu gewählten Regierung gegenüber liberalen Umbauagenten in Schlüsselpositionen zu stärken. Mithilfe von Notstandsverordnungen und einem Amtsenthebungsverfahren (sowie der demokratischen Legitimierung durch einen Volksentscheid) sollten der (neo)liberale und kontroverse Präsident Basescu, die von ihm und der liberalen Parlamentsmehrheit berufenen Richter des Verfassungsgerichts, der Ombudsmann und die Präsidenten der beiden Kammern des Parlaments entlassen werden. Dies war somit eine klassische Art von Politisierung staatlicher Institutionen mithilfe der Instrumentalisierung des Rechts. In Rumänien war dies eine gängige Praxis, die zuvor von liberalen und sozialdemokratischen Regierungen angewendet wurde, um ihre Macht zu stärken sowie um von der EUauferlegte Änderungen schnell, unproblematisch und teilweise undemokratisch durchführen zu können. ${ }^{68}$

Was der kritische und jeweilige Fortschrittsbericht vom Juli 2012 verschwieg, waren wichtige Hintergrundinformationen, um die Rechtsstaatlichkeitskrise aus einer zeitlichen, umfassenden und objektiven Perspektive beurteilen zu können:

63 Interview mit einem anonymen Justizrats-Mitglied. Siehe auch www.evz.ro/scandal-int re-sefii-magistratilor-judecatori-contra-procurori-horatius-dumbrava-csm-s-1.html (Zugriff vom 06.07.2016).

64 Europäische Kommission 2008, S. 12.

65 Siehe Mendelski 2011; Mendelski 2012; Mendelski 2015.

66 Europäische Kommission 2012.

67 Venice Commission, Opinion No. 685/2012, Strasbourg, 17. Dezember 2012.

$68 \mathrm{Zu}$ der Problematik der Gesetzgebung durch beschleunigte und außerplanmäßige Verfahren (zum Beispiel ohne ausführliche Deliberation in nationalen Parlamenten) im Rahmen der EU Erweiterung siehe Sadurski 2004; Risteska 2013; Goetz, Zubek 2007; Mendelski 2015. 
(1) Präsident Basescu (sowie Justizministerin Macovei) hatten in den Jahren zuvor das Justizsystem (einschließlich des Verfassungsgerichts und der Anti-Korruptionsbehörde) durch ihre eigenen subjektiven Nominierungen sowie die Instrumentalisierung des Rechts (unter stillschweigender Kenntnis der EU) politisiert. ${ }^{69}$

(2) Der Präsident war ein interventionistischer Akteur, welcher staatliche Strukturen instrumentalisierte beziehungsweise beeinflusste (Geheimdienst, Staatsanwaltschaft, Justizrat, Presse) und dadurch deren Unabhängigkeit missachtete sowie die Gewaltenteilung untergrub. ${ }^{70}$

(3) Der "unparteiische" Ombudsmann Gheorghe Iancu war zuvor von der liberalen PDL (der ehemaligen Partei des Präsidenten) vorgeschlagen worden. ${ }^{71}$

(4) Das Verfassungsgericht hat abusive Entscheidungen politischer Natur zum Amtsenthebungsverfahren des vom Volk delegitimierten ${ }^{72}$ Präsidenten erlassen, um die Amtsenthebung zu behindern (zum Beispiel Einführung eines Quorums von 50 Prozent, Verschiebung der Validierung des Referendums um einen Monat). ${ }^{73}$ Zudem wurde die nachträgliche Errata der Verfassungsentscheidung, welche eine neue, jeweilige Regel zur Berechnung des Quorums einführte und die Basescu vor der Amtsenthebung rettete, angeblich von Anhängern Basescus aus dem Justizrat entworfen und zudem ohne die Konsultation des gesamten Plenums im Verfassungsgericht. ${ }^{74}$ Eine politisch neutrale, systematische und zeitlich eingebettete Ana-

69 Interessanterweise wurde die Instrumentalisierung der Notstandsverordnungen durch die Regierung Tariceanu in Fortschrittsberichten unter dem Mandat Macoveis (2005-2007) überhaupt nicht erwähnt. Die EU hat auch keine Kritik geäußert, als die ehemalige Justizministerin die Kompetenz zur Ernennung des Generalstaatsanwalts und des Leiters der Anti-Korruptionsbehörde (DNA) vom Justizrat ins Justizministerium übertrug, um ihren eigenen Kandidaten den Vorzug zu geben. Das Gesetz Nr. 247/2005 wurde auch als "Macovei-Gesetz " bezeichnet, um die Jeweiligkeit und das persönliche Interesse hinter diesem Gesetz aufzuzeigen. Siehe www.criticatac.ro/22167/legea-maco vei-cronica-unei-dezamgiri-anunate/ (Zugriff vom 06.07.2016).

70 Interviews mit anonymen rumänischen Richtern. Siehe Ghiciusca 2007; Teodorescu, Sultanescu 2005; siehe das Interview mit dem ehemaligen Präsident Constantinescu für die tageszeitung vom 5. September 2012, www.constantinescu.ro/interviuri/interviuri_ 53.htm (Zugriff vom 06.07.2016).

71 Siehe www.pntcd.ro/gheorghe-iancu-vicepresedinte-pntcd-principiile-mele-sunt-profesi onalism-respect-si-smerenie-iar-acestea-se-regasesc-in-pntcd/; www.ziare.com/pdl/stiripdl/pdl-il-propune-pe-iancu-gheorghe-ca-avocat-al-poporului-1102337 (Zugriffe vom 06.07.2016).

7287 Prozent (acht Millionen) der im Referendum Votierenden haben sich für eine Amtsenthebung des Präsidenten ausgesprochen. Diese Mehrheit reichte laut Verfassungsgericht nicht aus, um das Quorum von 50 Prozent zu erreichen, was auf Basis einer veralteten Volkszählung kalkuliert wurde und die drei Millionen ausgewanderten Rumänen nicht berücksichtigte.

73 Siehe www.constantinescu.ro/interviuri/interviuri_53.htm (Zugriff vom 06.07.2016).

74 Siehe www.antena3.ro/politica/sinteza-zilei-fost-membru-csm-consiliul-a-scris-erata-ca re-l-a-salvat-pe-traian-basescu-295317.html; http://jurnalul.ro/stiri/observator/tudorel-t oader-judecator-ccr-am-solicitat-presedintelui-convocarea-de-urgenta-a-plenului-curtii620593.html; www.cotidianul.ro/ccr-a-violat-legea-referendumului-190703/ (Zugriffe vorn 26.07.2016). 
lyse der Rechtsstaatlichkeit hätte also zu dem Schluss kommen müssen, dass die sogenannten "willentlichen Aktionen " der Regierung Ponta nur eine politische, vom Volk legitimierte Gegenreaktion auf die zuvor erfolgte Politisierung und Instrumentalisierung des Präsidenten sowie der Regierung Tariceanu waren. Der einseitige Bericht der Europäischen Kommission hat jedoch nur das Verhalten der PSD-Regierung kritisiert und damit viel Kritik seitens rumänischer und internationaler Beobachter hervorgerufen. ${ }^{75}$ Unter den lautstarken rumänischen Kritiken waren zum Beispiel die öffentlichen Briefe von Stelian Nastase und unabhängigen Wissenschaftlern, ${ }^{76}$ die Kritik des früheren und kürzlich verstorbenen Verfassungsrichters Ciobanu, welcher die nichtobjektive und intransparente Methodologie der Kommission anprangerte, ${ }^{77}$ sowie die Kritik des ehemaligen (liberalen) Präsident Emil Constantinescu, welcher die Europäische Kommission anklagte, eine parteiische, irreführcnde und faktisch falsche Evaluierung veröffentlicht $z$ u haben. ${ }^{78}$

Ist der hier präsentierte Fall Rumäniens ein Ausnahmefall? Weitere detaillierte Fallstudien und Untersuchungen wären nötig, um eine systematische Politisierung festzustellen. Allerdings gibt es Anzeichen dafür, dass Rumänien kein Einzelfall ist. Frühere Studien haben bereits auf die nichtobjektive und defizitäre EU-Evaluierungsmethodik im Falle ehemaliger Beitrittsländer, ${ }^{79}$ des Mitgliedslands Bulgarien ${ }^{80}$ sowie der Nachbarländer ${ }^{81}$ hingewiesen. In diesem Zusammenhang könnte man auch den nichtobjektiven Bericht des Europäischen Parlaments nennen (zum Beispiel den Tavares-Bericht), welcher der Orban-Regierung in Ungarn vorwarf, das Recht (Verfassung, Kardinalgesetze), die Medien und die Justizstrukturen zu instrumentalisieren/politisieren und die Verfassungskontrollen (checks \& balances) auszuhöhlen, jedoch ähnliche (beziehungsweise strukturelle) Praktiken und Vergehen der Horn-Regierung (1994-1998) und der Gyurcsany-Regierung (2004-2009) nicht erwähnte. Die einseitige Reaktion der EU wurde daher von EU-Parlamentariern als

75 Siehe http://actmedia.eu/daily/heated-debates-among-mep-groups-over-the-political-sit uation-in-romania/42025_(Zugriff vom 06.07.2016).

76 Siehe www.stelian-tanase.ro/scrisoare-catre-uniunea-europeana-bruxelles/ (Zugriff vom 06.07.2016).

77 Siehe www.juridice.ro/221992/din-nou-despre-obstructionarea-justitiei-intrarea-in-vig oare-a-noului-cod-de-procedura-civila-atributiile-consiliului-superior-al-magistraturii-s i-rolul-curtii-constitutionale.html (Zugriff vom 06.07.2016).

78 Siehe www.nineoclock.ro/the-report \%E2\%80\%99s-dangerous-omissions; www.nineo clock.ro/former-president-emil-constantinescu-writes-to-ec-president-jose-manuel-barr oso/ (Zugriffe vom 06.07.2016).

79 Kochenov 2008.

80 Dimitrov et al. 2014; Toneva-Metodieva 2014.

81 Schimmelfennig 2012. 
Inkonsistenz und Doppelmoral kritisiert. ${ }^{82}$ Zudem wurden die meisten Vorwürfe des Tavares-Berichts in einem offenen Memorandum von der ungarischen Regierung als ungenau, faktisch unwahr und parteiisch zurückgewiesen. ${ }^{83}$ Zweifellos ging die Orban-Regierung bei einigen Entscheidungen an die Grenze der Legalität, aber wo diese Grenze übertreten wurde (wie zum Beispiel im Falle der frühzeitigen Pensionierung und des Transfers von Richtern), ruderte die Regierung mehrfach zurück und übernahm Gerichtsentscheidungen des Verfassungsgerichts sowie des Europäischen Gerichtshofs.

Insgesamt ergibt sich also das Bild, dass mit zweierlei Maß gemessen wird, je nachdem welche Parteien an der Macht sind beziehungsweise welche Personen wichtige Positionen in den Staatsstrukturen besetzen. Die einseitige, parteiische Evaluierung spiegelt sich auch in den »rhetorischen Aktionen« der EU wider.

\subsection{Parteiliches »rhetorisches Handeln« während der Verfassungskrisen}

Es gibt einige konkrete Hinweise darauf, dass die EU (sowohl Vertreter der Kommission als auch des Parlaments) während der politischen Machtkonflikte (der sogenannten "Verfassungskrisen «) in Rumänien, Ungarn und Polen zu parteiischer, konfliktschürender Rhetorik gegriffen hat und bei ähnlichen Rechtsstaatlichkeitsvergehen durch EU-freundliche Regierungen davon abgesehen hat. Dieses parteiliche »rhetorische Handeln « der EU reflektiert ein nichtsystematisches Bewerten und Sanktionieren von Rechtsstaatlichkeitsvergehen innerstaatlicher Akteure. Es zeigt sich zunächst einmal während der Verfassungskrise in Rumänien, bei der die EU durch ihre einseitige Rhetorik in den innerstaatlichen Machtkonflikt zwischen Ministerpräsident Ponta und den liberalen Umbauagenten (Präsident Basescu, Richter des Verfassungsgerichts und Ombudsmann) eingriff.

Die sogenannte rumänische Verfassungskrise vom Juli 2012 resultierte nicht nur in einen parteiischen Fortschrittsbericht der Kommission, sondern auch in einer aufgeheizten, mit aggressiver Rhetorik geführten öffentlichen Debatte. So interpretierten bekannte EU-Parlamentsabgeordnete (Elmar Brok, Joseph Daul, Monica Macovei) die "willentlichen Aktionen « der PSD-Regierung unter Victor Ponta vom Sommer 2012 als »coup d'etat«. ${ }^{84}$ Ähnlich äußerte sich die damalige Justizkom-

82 Siehe www.politics.hu/20130509/top-fideszers-complain-of-bias-errors-double-standar ds-in-ep-hungary-report; siehe auch die Plenardebatten des Europäischen Parlaments vom 12. September 2012 ("Politische Lage in Rumänien ". http://goo.gl/Iw4ES6) und vom 19. Januar 2016 ( Situation in Polen «. http://goo.gl/kuoSXg); außerdem die Kommentare von Frank Engel und Kinga Gal zum EP LIBE-Komitee (Civil Liberties, Justice and Home Affairs). Siehe auch "The situation of fundamental rights in Hungary«. www.youtube.com/watch?v=kaMkcz $2 \mathrm{z} 8 \mathrm{mQ}$; www. youtube.com/watch? $\mathrm{v}=\mathrm{v} 4 \mathrm{AKnDJq}$ FE8 (Zugriffe vom 06.07.2016).

83 Es lohnt sich hier, das ausführliche und kaum bekannte Memorandum der Regierung zu lesen. Siehe Remarks of the government of Hungary on the report of the European Parliament on the situation of fundamental rights in Hungary. http:/goo.g1/3ruyys (Zugriff vom 06.07.2016).

84 Siehe www.eppgroup.eu/fr/press-release/Roumanie\%3A-situation-politique-de-coup-d' $\%$ C3\%89tat (Zugriff vom 06.07.2016). 
missarin Vivianne Reding, die von »einer Art Putsch des Parlaments « 85 und einer "Rechtsstaatlichkeitskrise " sprach. ${ }^{86}$ Auf der anderen Seite bewerteten einige Parlamentsabgeordnete (zum Beispiel Hannes Swoboda, Graham Watson, Renate Weber, Pino Arlacchi) diese rhetorischen Vorwürfe als unbegründet und falsch. ${ }^{87}$ Der ehemalige rumänische liberale Präsident Constaninescu kritisierte diesen thetorischen Druck der EU als " unreal accusations that are contradicted by any correct and unbiased analysis of events and documents" .88 Auch wenn es für neutrale Außenstehende schwer ist, die Legitimität und Legalität der streitenden Parteien festzustellen, so wird zumindest klar, dass EU-Vertreter im Fall Rumäniens mit ihrer scharfen, delegitimierenden Rhetorik zu weit gegangen sind.

Ähnliche externe und rhetorische Delegitimierungsstrategien durch liberale EUVertreter (trotz der durch den demos verliehenen internen Legitimität) finden wir einige Jahre später im Falle der polnischen "Verfassungskrise" aus dem Jahr 2015/2016. Die parteiische Rhetorik der EU kam wieder zum Vorschein während des Machtkampfs zwischen dem Verfassungsgericht (und im Besonderen dem von der liberalen PO-Partei nominierten Verfassungspräsidenten Rzeplinski) und Vertretern der konservativen PIS-Partei (Kaczynski) sowie dem polnischen Präsidenten Duda, einem ehemaligen PIS-Mitglied. ${ }^{89}$ Dieser Konflikt begann im Juni 2015, als es der Vorgänger-Regierung (PO) mit einer umstrittenen Vorschrift gelang, fünf neue Richter und damit 14 von 15 Richtern des Verfassungsgerichts zu bestimmen und damit das Gericht vollständig zu kontrollieren. Trotz dieser fraglichen Aktion intervenierte die EU nicht, auch weil kaum Alarmglocken der transnationalen Netzwerke läuteten. Die neu gewählte, konservative PIS-Regierung sowie Präsident Duda wollten diese unübliche konstitutionelle Praxis und den Machtverlust im Ver-

85 Siehe www.lemonde.fr/europe/article/2012/09/01/viviane-reding-meme-en-crise-l-europ e-n-est-pas-qu-un-marche_1754411_3214.html (Zugriff vom 06.07.2016). Interessanterweise ist die kontroverse Aussage Redings nicht mehr auf der Webseite der führenden französischen Zeitung Le Monde abrufbar. Allerdings kann das Interview Redings für Le Monde hier nachgelesen werden: www.scoop.it/t/l-europe-en-questions/?tag=Hongr ie sowie auf Rumänisch unter http://revistapresei.hotnews.ro/stiri-subiectele_zilei-1313 8266-viviane-reding-nu-surprinsa-amanare-aderarii-romaniei-schengen.htm (Zugriffe vom 06.07.2016).

86 Siehe www.europeanvoice.com/article/imported/the-romanian-coup-d-ctat/74831.aspx; http://europa.eu/rapid/press-release_SPEECH-13-677_de.htm (Zugriffe vom 06.07.2016).

87 Siehe www.europarl.europa.eu/sides/getDoc.do?pubRef=-//EP//TEXT+CRE+20120912 +ITEM-011+DOC+XML+V0//EN (Zugriff vom 06.07.2016).

88 Siehe http://tolerance.ro/portal/rule-of-law/former-romanian-president-emil-constantin escu-to-ep-president-martin-schulz-basescus-mandate-is-ille/; www.nineoclock.ro/the-r eport \%E2\%80\%99s-dangerous-omissions (Zugriffe vom 06.07.2016). Siehe auch den Brief des ehemaligen Präsidenten Constantinescu an die Europäische Kommission: www.nineoclock.ro/former-president-emil-constantinescu-writes-to-ec-president-josemanuel-barroso/; www.dcnews.ro/212803_212803.html (Zugriffe vom 06.07.2016).

89 Siehe www.telegraph.co.uk/news/worldnews/europe/poland/12051752/EU-parliamenthead-refuses-to-a pologise-over-coup-comment-after-Polish-PM-request.html (Zugriff vom 06.07 .2016$)$. 
fassungsgericht nicht einfach so hinnehmen und verabschiedeten kurzfristig Gesetze, die das Verfassungsgericht pluralistischer machen sollten, dabei aber die Unabhängigkeit des Verfassungsgerichts untergruben. Wieder einmal schaltete sich die EU mit scharfer, nichtobjektiver Rhetorik ein. Diesmal sprach EU-Parlamentspräsident Martin Schulz in einem Interview vom »Staatsstreich-Charakter " ${ }^{90}$ und von "gelenkter Demokratie nach Putins Art", was Unverständnis und kritische Reaktionen seitens der polnischen Regierung auslöste sowie Anlass für mehrere offene Briefe war. Die frühere niederländische EU-Justizkommissarin Viviane Reding (jetzt EVP-Abgeordnete) sprach in einem Interview vom $»$ Angriff auf den Rechtsstaat " und von "Putin-Orbán-Kaczynski-Logik ". ${ }^{91}$ Die polnische Regierung wies diese Anschuldigungen als »unakzeptabel «, »unbegründet « und »unverantwortlich « zurïck und bekräftigte, dass die Demokratie, der Rechtsstaat sowie die Pressefreiheit in Polen nicht gefährdet scicn. ${ }^{92}$

Die kritische, unangebrachte EU-Rhetorik war (ähnlich wie im Falle Rumäniens) parteiisch und nicht konsensfördernd. Zunächst ist das polnische Verfassungsgericht (trotz seines relativ hohen Ansehens) eine nicht ideologiefreie Institution ${ }^{93}$ und wurde sowohl von liberalen als auch von konservativen Parteien durch parteiliche Nominierungen politisiert (wenn auch nicht so stark wie in Rumänien). Dabei trugen das politische Verhalten und Aussagen des Verfassungspräsidenten Rzeplinski nicht unbedingt zur Entpolitisierung des Gerichts bei. ${ }^{94}$ Objektive Kenner der Rechtsstaatlichkeit in Polen wiesen darauf hin, dass sowohl die Vorgänger-Regierung der $\mathrm{PO}$ als auch die PIS-Regierung das Verfassungsgericht jüngst durch »rechtlich und moralisch zweifelhafte "Besetzungen politisierten, welche in der 30-jährigen Geschichte des Verfassungsgerichts bisher nicht vorgekommen waren. Die Folgen dieses politischen Machtkampfs waren eine Verschärfung des Stellvertreterkonflikts zwischen dem entmündigten, größtenteils PO-nominierten Verfassungsgericht und der unnachgiebigen PIS-Regierung sowie ein »Ruinieren der politischen und verfassungsrechtlichen Kultur.$^{95}$ Die Venedig-Kommission bestätigte zwar Rechtsstaatlichkeitsverstöße der PIS-Regierung, sprach aber gleichzeitig von problematischem Verhalten der Vorgängerregierung und forderte einen politischen

90 Siehe www.deutschlandfunk.de/regionalwahlen-in-frankreich-frau-le-pen-ist-wahlpoliti sch.694.de.html?dram:article_id=339718 (Zugriff vom 06.07.2016).

91 Siehe www.faz.net/aktuell/politik/ausland/europa/martin-schulz-greift-polnische-politik -an-14005694.html (Zugriff vom 06.07.2016).

92 Siehe www.rt.com/news/339524-european-parliament-comdemn-poland/ (Zugriff vom 06.07.2016).

93 Kantorowicz, Garoupa 2016. Man bedenke auch, dass die meisten Richter des liberalen Verfassungsgerichts ehemalige DAAD- oder Humboldt-Stipendiaten sind.

94 Siehe www.tvp.info/25487033/prof-rzeplinski-jest-bardziej-politykiem-niz-sedzia; http://wpolityce.pl/polityka/290796-prof-andrzej-rzeplinski-robi-polityke-w-coraz-mni ej-zawoalowany-sposob-wiklo-i-pyza-we-wsieci-o-politycznej-karierze-prezesa-trybuna lu-konstytucyjnego (Zugriffe vom 06.07.2016).

95 Radwan 2016, S. 12. 
Kompromiss. ${ }^{96}$ Insgesamt bleibt also unverständlich, warum die EU einerseits Regelbrüche einer (konservativen) Partei heftig kritisierte und gegen sie polemisierte ${ }^{97}$ und andererseits diese bei einer anderen (liberalen) Partei nicht monierte. Als ein weiteres Beispiel kann man anführen, dass es im Falle Tschechiens zu keiner "coup d «etat«-Rhetorik der EU kam, als dort der euroskeptische Präsident Vaclav Klaus aufgrund seiner kontroversen Begnadigungen seines Amtes enthoben werden sollte. ${ }^{98}$ Wie lässt sich diese Doppelmoral (und das inhärente Evaluierungsdefizit) erklären? Kann man die Ursachen des europäischen Evaluierungsdefizits benennen?

\section{Diskussion: Potenzielle Ursachen für das EU-Evaluierungsdefizit}

\subsection{Problembezogene Evaluierung: Fehlen einer konsistenten und systematischen Methodologie}

Eine wichtige Ursache für das EU-Evaluierungsdefizit ist das Fehlen einer systematischen, umfassenden Methodik und das Vorherrschen eines "problembezogenen " Ansatzes. Der problembezogene, reaktive (und nicht proaktive) Ansatz bedeutet, dass die EU auf neu auftretende Rechtsstaatlichkeitsprobleme in Beitritts- und Mitgliedsländern nur punktuell reagiert, wenn "Alarmglocken « der internationalen Rechtsgemeinschaft, der liberalen Presse sowie der vom Westen finanzierten Nichtregierungsorganisationen läuten. ${ }^{99}$ Das heißt, erst wenn genug "Krach « (beziehungsweise moralischer Druck) durch transnationale Netzwerke ${ }^{100}$ gemacht wird, mobilisiert die EU und kritisiert eventuelle Rechtsstaatlichkeitsvergehen in offiziellen Mitteilungen, Fortschrittsberichten der Kommission oder neuerdings auch in öffentlichen Debatten im Europäischen Parlament, in denen die jeweiligen Mitgliedstaaten an den Pranger gestellt werden (" naming and shaming-Debatten «). Die EU, welche keine eigene profunde Kompetenz hat, die Rechtsstaatlichkeit zu beurteilen (im Gegensatz zur Venedig-Kommission), war und bleibt somit weiterhin abhängig von der »Kompetenz " einer gut vernetzten, legalen und liberalen, epistemischen Experten-Gemeinschaft (inklusive NGOs), die tendenziell viel weniger Druck ausübt und kritisiert, wenn änderungswillige liberale Eliten die Rechtsstaatlichkeit mit Füßen treten. ${ }^{101}$ Diese Problematik, welche die Objektivität zur Beurteilung der Rechtsstaatlichkeit untergräbt, tritt im Übrigen auch auf, wenn man

96 Venice Commission 2016, S. 18.

97 Hans Olaf Henkel sprach von einer "grotesken Inquisition « der polnischen Ministerpräsidentin Szydlo (statt von einem Dialog mit ihr), als eine Debatte im Europäischen Parlament zu der Verfassungskrise in Polen stattfand. Siehe www.europarl.europa.eu' sides/getDoc.do?type=CRE\&reference $=20160119 \&$ secondRef $=I T E M-010 \&$ languag $e=L V$ (Zugriff vom 06.07.2016).

98 Siehe www.eazi.ro/prin-lume/atentie-barroso-reding-gordon-gitenstein-lovitura-de-st at-in-cehia (Zugriff vom 06.07.2016).

99 Interview mit einem anonymen Repräsentanten der Europäischen Kommission.

100 Siehe Risse, Sikking 1999.

101 Siehe Parau 2015. 
liberale NGO-Vertreter (Bertelsmann Stiftung, Freedom House) als Experten für Evaluationsberichte zur Rechtsstaatlichkeit hinzuzieht. ${ }^{102}$

Die problemorientierte, reaktive Natur der EU hat einen entscheidenden Mangel. Die EU-Kommission reagiert auf "systematische Rechtsstaatlichkeitsvergehen * (zum Beispiel Missbrauch des Rechts, Politisierung etc.) erst dann, wenn die internationalen "Alarmglocken « läuten. ${ }^{103}$ Die Frage, die man sich stellen sollte, ist: Warum schreien die meist selbsternannten internationalen Wächter der Rechtsstaatlichkeit (und ebenso die EU) nur zu bestimmten Zeiten beziehungsweise bei bestimmten "illiberalen « Akteuren auf, obwohl es sich in den meisten Fällen um systematische Probleme der Rechtsstaatlichkeit handelt, die mit der politischen und konstitutionellen Kultur zusammenhängen?

Die EU hat großen Spielraum für ihre kritische Reaktion, und es wird deutlich, dass systematische Rechtsstaatlichkeitsvergehen eher geahndet werden, wenn »nichtliberale (das heißt beispielsweise national-konservative oder russlandorientierte) Eliten die Regierung übernehmen, liberale, prowestliche Eliten dagegen geschont werden, auch wenn sie ihrerseits die Rechtsstaatlichkeit untergraben. Beispiele für nicht geahndete systematische Rechtsstaatlichkeitsverletzungen von liberalen Umbauagenten stammen aus Rumänien (wo Justizstrukturen und Gesetze von Präsident Basescu und Ministerin Macovei politisiert und instrumentalisiert wurden), aus Ungarn (wo bereits die Gyurcsany-Regierung die Justizunabhängigkeit und die Verfassung untergrub) und aus Polen (wo die liberale Bürgerplattform PO unter Kopacz das Verfassungsgericht politisierte). Ähnliche unsystematische Evaluierungen der Rechtsstaatlichkeit sind in postsowjetischen Ländern zu finden (zum Beispiel Ukraine, Moldawien und Georgien). Dass es zu solch einer einseitigen und inkonsistenten Evaluierung seitens der EU kommt, hängt unter anderem eben mit der einseitigen Auswahl von Informationsquellen zusammen, auf die sie sich stützt.

\subsection{Informationsasymmetrie: Eine vorwiegend liberal-konstitutionelle Perspektive}

Die EU-Evaluierung der Rechtsstaatlichkeit leidet also unter einer erheblichen Informationsasymmetrie, die dazu führt, dass Rechtsstaatlichkeit aus einer einseitigen, vornehmlich liberal-konstitutionellen Perspektive betrachtet wird. Dies ist zunächst einmal dem Screening-Prozess geschuldet, das heißt dem Sammeln von Informationen über den Zustand der Rechtsstaatlichkeit in einem Land. Das Hauptproblem ist hierbei, dass EU-Beobachter, Berichterstatter und Gutachter tendenziell Personen aus dem liberalen Lager treffen, dic genannten "Umbauagenten “ aus den neu geschaffenen Institutionen, die die EU in ihren Berichten lobt und finanziell fördert (zum Beispiel Verfassungsgerichte, Anti-Korruptions- und Integritätsbehörden, Justizräte, spezielle Regulationsbehörden), sowie EU-Diplomaten und liberale Reprä-

102 Als unabhängiger Zweitgutachter für die Bertelsmann Stiftung konnte ich erleben, wie ein nationaler Erstgutachter (Vertreter einer NGO) - ähnlich wie die EU - alle Reformbemühungen der liberalen "change agents « und neu geschaffenen Strukturen positiv beurteilte, ohne dabei die politisierten und instrumentalisierten Prozesse und Aktionen zu kritisieren.

103 Ähnlich reaktiv agiert die Venedig-Kommission. 
sentanten von NGOs. Im Grunde besteht ein Großteil des Informationsnetzwerks aus Anhängern des dominanten liberalen Paradigmas (das heißt der konstitutionellen Demokratie, des liberalen Konstitutionalismus und der Justizialisierung). ${ }^{104}$ Ein kurzer Blick auf die Lebensläufe der Justiz- und Anti-Korruptionseliten (inklusive der Verfassungsrichter) aus Osteuropa zeigt, dass diese liberalen »change agents « zunehmend in epistemischen Gemeinschaften der transnationalen Netzwerke ausgebildet und sozialisiert wurden (zum Beispiel während diverser Seminare der Europäischen Rechtsakademie in Trier, des Europarats in Strasbourg oder von EU-Institutionen in Brüssel und Luxembourg (EU) als auch in der OECD in Paris). ${ }^{105}$ Es ist klar, dass viele dieser entsprechend "sozialisierten " (teilweise indoktrinierten) Umbauagenten wenig geneigt sind, ihre ausländischen "Sponsoren " zu kritisieren sowie die internationalen »best standards « zu hinterfragen. Dies gilt im Übrigen auch für die Vertreter von finanziell abhängigen NGOs und Regierungen, die es oft nicht wagen, die Strategien und Projekte ausländischer Förderer zu hinterfragen. ${ }^{106}$ Zudem sollte berücksichtigt werden, dass auf unkritische und "loyale« inländische Eliten eine mögliche Karriere in Brüssel oder internationalen Organisationen wartet. Insgesamt ist es also fraglich, ob ein objektiver Informationspluralismus (und damit eine unparteiische Bewertung) der Rechtsstaatlichkeit überhaupt garantiert werden kann.

Zusätzlich sollte man berücksichtigen, dass die sogenannten "unabhängigen " Experten und Berater der EU teilweise eng mit EU-Institutionen verbunden sind, zum Beispiel in Form von früherer Beschäftigung und Zusammenarbeit sowie als (ehemalige) Mitglieder in transnationalen, liberalen (Justiz-)Netzwerken, was wiederum potenzielle Interessenkonflikte generieren und die unparteiische Evaluierung der Rechtsstaatlichkeit beeinflussen kann. Ein kurzer Blick auf die Lebensläufe der Berater der Europäischen Kommission zeigt die Plausibilität dieses Arguments. Die meisten Sonderberater der Kommissionäre für Justiz (Reding und Timmermans) sind entweder ehemalige Mitarbeiter der EU (Kommission, Gerichtshof) oder eingebunden in liberale, transnationale Netzwerke (zum Beispiel Aspen Institute, European Council on Foreign Relations, World Bank, World Economic Forum, Justiznetzwerke). ${ }^{107}$ Die einseitige Abhängigkeit von liberalen, EU-verbundenen Experten muss in dieser Häufung notwendigerweise die Objektivität der Beurteilung der Rechtsstaatlichkeit beeinflussen. Dies ist yor allem dann kritisch zu sehen, wenn man Rechtsstaatlichkeit als ein politisch, sozial und historisch eingebettetes Prinzip betrachtet. ${ }^{108}$

104 Interview mit einem anonymen internationalen Rechtsstaatlichkeitsexperten.

105 Ich hatte mehrmals selbst die Gelegenheit gehabt, hochrangige Richter und Staatsanwälte bei internationalen Fortbildungsseminaren und Konferenzen kennenzulernen (zum Beispiel in Trier, in Strasbourg, Luxembourg und in Brüssel).

106 Interview mit einem Richter des Europäischen Gerichtshofs für Menschenrechte (EGMR); siehe Carothers 2003; Channell 2006.

107 Siehe http://ec.europa.eu/civil_service/about/who/sa_en.htm (Zugriff vom 06.07.2016).

108 Carrerra et al. 2013; Mendelski 2014. 
Der Fall der Rechtsstaatlichkeits-Mission der EU im Kosovo (EULEX) spiegelt die Problematik der sogenannten "unabhängigen " Experten in aktueller Weise wider. Nachdem Maria Bamieh (die für EULEX arbeitete) auf den Missstand der korrupten EULEX-Staatsanwälte hinwies, beauftragte die Hohe Vertreterin der EU für Außenund Sicherheitspolitik, Federica Mogherini, den »unabhängigen« Experten Prof. Paul Jacque, den Umgang der EULEX-Führung mit den Korruptionsvorwürfen zu überprüfen. ${ }^{109}$ Der von Prof. Jacque verfasste Bericht vom 30. März 2015 hat die Vorwürfe zurückgewiesen, dass EULEX versucht habe, die Korruption seiner Beamten zu vertuschen, ebenso wie den Vorwurf, dass die Informantin aufgrund ihrer Enthüllungen schikaniert wurde. ${ }^{110}$ Die Untersuchungen des Korruptionsskandals sind noch nicht abgeschlossen, aber die Ernennung des »unabhängigen « Gutachters Jacque ist kritikwürdig. Prof. Jacque war zwischen 1992 und 2008 der Direktor im Juristischen Dienst des Europäischen Rats und arbeitete somit für eine Institution, die das EULEX-Mandat verfasste. Zudem evaluierte er ein paar Jahre später selbst. ${ }^{111}$ Es stellen sich also an diesem Punkt eine Reihe von Fragen zur Unabhängigkeit der von der EU benannten Experten, die einer systematischen Analyse unterworfen werden müssen.

\subsection{Zusätzliche Beeinflussungs- und Manipulationsmöglichkeiten}

Selbst wenn wir optimistisch sind und annehmen, dass die meisten EU-Experten keine Interessenkonflikte haben und unabhängig sind, könnten zusätzliche Faktoren im Evaluierungsprozess eine Rolle spielen, die in einer einseitigen Information und damit einer nichtobjektiven Evaluierung der Rechtsstaatlichkeit resultieren.

Erstens gibt es Möglichkeiten des politischen und diplomatischen Einflusses auf den Inhalt der Fortschrittsberichte seitens des Rates (in Form von bilateralem Druck mächtiger Staaten), seitens des Sekretariats der Europäischen Kommission und seitens der Diplomaten aus den jeweiligen EU-Delegationen vor Ort. ${ }^{112}$ Als Beispiel fehlender Transparenz des Evaluierungsprozesses sei nur an die parteiliche Evaluation der Rechtsstaatlichkeit durch die EU-Delegationen erinnert, die im Falle ehemaliger Sowjetrepubliken (Moldawien, Ukraine und Georgien) offensichtlich wurde. So wurden in Moldawien änderungsbereite, jedoch fragwürdige Eliten der Allianz für Europäische Integration (AIE) von der EU und US-Repräsentanten trotz ihres Missbrauchs des Rechts, der Grundrechte und der Politisierung der Justiz unterstützt, und ihre Arbeit wurde als Erfolg (success case) evaluiert. Auch wenn Probleme mit Rechtsstaatlichkeit bemerkt wurden, erlaubte die EU ihren ände-

109 Siche http://eeas.europa.eu/statements-eeas/2014/141110_02_en.htm (Zugriff vom 06.07.2016).

110 Der Report von Prof. Jacque ist hier abrufbar: http://eeas.europa.eu/statements-eeas/ docs/150331_jacque-report_en.pdf (Zugriff vom 06.07.2016).

111 Siehe https://euobserver.com/investigations/126468 (Zugriff vom 06.07.2016).

112 Interviews mit einem ehemaligen Diplomaten und Regierungsberater. 
rungswilligen Umbauagenten sogenannte »Honeymoon «-Übergangsperioden nach dem Machtwechsel 2009.113

Während dieser Übergangsperioden wurde den reformbereiten, prowestlichen Eliten der AIE eine "carte blanche" aufgrund von »Deals zwischen Freunden « ausgestellt. ${ }^{114}$ Das stille, nichtkritische Verhalten der EU hat dann die Ansammlung und den Missbrauch der politischen Macht in wenigen Händen von prowestlichen Oligarchen gefördert, zum Schaden der Rechtsstaatlichkeit (im Besonderen der Gewaltenteilung). Fehlende interne und externe Rechenschaftspflichten resultierten in politisierten Regierungs- und Reformmethoden, in zahlreichen skandalösen Affären unter Missbrauch von Justizstrukturen ${ }^{115}$ sowie schrumpfender Legitimität gegenüber der Bevölkerung. Die frühere übertriebene (da parteiische) Bewertung Moldawiens als "success story "wurde kürzlich von politischen Analysten revidiert. Mittlerweile wird das frühere Vorzeigeland Moldawien als »Misserfolg der Östlichen Partnerschaft « bewertet. ${ }^{116}$ Ein ähnliches Bild von Macht- und Rechtsmissbrauch, Politisierung von Justiz und Anti-Korruptionsreformen durch prowestliche Akteure sowie inkonsistenter Bewertung der Rechtsstaatlichkeit (inklusive Menschenrechte) durch die EU ergibt sich auch für die Übergangsperioden nach der Orangenen Revolution (2004) und dem Euromaidan (2013) in der Ukraine sowie für die Amtsperiode unter Präsident Shakashvilli in Georgien. ${ }^{117}$ Das EU-Evaluierungsdefizit spiegelt sich ebenso in dem jüngsten EULEX-Korruptionsskandal im Kosovo wider, wobei informierte Kenner der EULEX-Führung Untätigkeit bei der Verfolgung von hochrangigen korrupten Regierungsmitgliedern ${ }^{118}$ und korrupten EULEX-Mitarbeitern vorwarfen. ${ }^{119}$

Wie kann man diese inkonsistente, parteiische Bewertung der Rechtsstaatlichkeit erklären? Ein möglicher Grund könnte eine Veränderung der politischen Machtkonstellation zugunsten von Umbaubefürwortern sein, die bestimmte wirtschaftliche, geopolitische und ideologische Interessen durch (neo)liberale Reformen erreichen sollen. ${ }^{120}$ Liberale Vertreter der EU möchten also zunächst EU-freundliche Eliten (zum Beispiel prowestliche ukrainische, georgische und moldawische Oligarchen) als demokratische, Rechtsstaatsprinzipien respektierende, liberale Eliten darstellen, um deren Chancen bei politischen Wahlen zu erhöhen. Gleichzeitig wer-

113 Interview mit einem anonymen Repräsentanten der EU-Delegation in Chisinau.

114 Siehe Belloni, Strazzari 2014.

115 Beispielsweise diverse Korruptionsskandale, Verbote von konkurrierenden, prorussischen Medien und Parteien, betrügerische Übernahmen von Unternehmen und Banken, Geldwäsche in Milliardenhöhe und der Raub einer Milliarde US-Dollar.

116 Kostanyan 2016; www.moldova.org/en/moldova-eus-failed-success-story/; www.hot news.ro/stiri-opinii-20709654-analiza-republica-moldova-cosmarul-continua.htm (Zugriffe vom 06.07.2016).

117 Di Puppo 2010; siehe auch Börzel, Pamuk 2012.

118 Capussela 2015.

119 Siehe http://derstandard.at/2000007908606/Kosovo-Eulex-Staatsanwaeltin-zieht-geg en-EU-Mission-ins-Feld (Zugriff vom 06.07.2016).

120 Siehe Börzel, Hüllen 2014. 
den die nichtliberalen, national oder nach Osten orientierten Oligarchen strenger bewertet, um diese in den Augen der Wähler zu diskreditieren. Die negative Folge ist dann oft eine Verringerung der Rechenschaftspflicht der extern legitimierten und gestärkten Eliten, was wiederum zu Missbrauch der Macht und des Rechts führt.

Zweitens kann es bereits während des Beobachtungsprozesses (Screening) zu Fehleinschätzungen der Rechtsstaatlichkeit kommen. Die nichttransparente und unsystematische Art der Zusammenstellung von Informationen aus verschiedenen Quellen (Expertenberichten) kann zum Beispiel in Über- beziehungsweise Unterbewertungen von Rechtsstaatlichkeitsproblemen resultieren, sogar wenn relativ unabhängige Experten und Beobachter (zum Beispiel OECD, SIGMA, GRECO, NGOs) detaillierte und vergleichsweise objektive Vorberichte verfassen. Das Weglassen von kritischen Informationen aus OECD-, SIGMA- und GRECO-Berichten in den Bereichen Governance und Korruption war zum Beispiel eine gängige Praxis der EU bei der Evaluierung Rumäniens während der Heranführungsphase zur EU-Mitgliedschaft. ${ }^{121}$

Drittens könnte die Wahrnehmung der Rechtsstaatlichkeit in einem Land von den politischen Netzwerken im Europäischen Parlament sowie von justiziellen Netzwerken (sowohl positiv als auch negativ) beeinflusst werden. Die jüngsten Beispiele von parteiischer Rhetorik in Berichten von engagierten liberalen Parlamentsabgeordneten (zum Beispiel Schulz, Tavares etc.) stammen aus $\gg$ Debatten « (die eher inquisitorischen Anklagen gleichen) zu den politisch-konstitutionellen Krisen in Rumänien, Polen und Ungarn. Schließlich gibt es transnationale justizielle ExpertenNetzwerke, ${ }^{122}$ die aus Richtern, Staatsanwälten und liberalen Akademikern bestehen, die durch ihre einseitige, legal-konstitutionelle Perspektive auf Rechtsstaatlichkeit nur ausgewählte Rechtsstaatsproblematiken ansprechen (wie zum Beispiel die Unabhängigkeit der Verfassungsgerichte und Justizräte) und die Rechenschaftspflicht dieser Organe kaum erwähnen. Zudem ist zu bedenken, dass die von der EU genutzten justiziellen Experten-Netzwerke ${ }^{123}$ aus hochrangigen Vertretern von Justizräten bestehen, welche in Osteuropa von der EU zuvor finanziell gefördert wurden, und aus Vertretern der Hohen Gerichte, die vor allem in Südosteuropa weiterhin stark politisiert sind. ${ }^{124}$

Insgesamt besteht also eine Vielzahl von mehr oder weniger sichtbaren Beeinflussungsmöglichkeiten der europäischen Evaluierungsmethodik der Rechtsstaatlichkeit. Die potenzielle Einseitigkeit, die konfliktschürende Rhetorik und die parteiische Bewertung der Rechtsstaatlichkeit in Berichten sind im Endeffekt schädlich für Demokratie und Rechtsstaatlichkeit, weil durch die fehlende Kritik von EUfreundlichen Eliten (sowohl in der Politik als auch in der Justiz) die externe und die

121 Interviews mit anonymen GRECO-, OECD- und SIGMA-Repräsentanten.

122 Parau 2015; Piana, Dallara 2015.

123 Dazu gehören das Netzwerk der Präsidenten der obersten Gerichtshöfe der EU, der Vereinigung der Staatsräte und der obersten Verwaltungsgerichte der EU oder der Richterräte; http://eur-lex.europa.eu/legal-content/DE/TXT/?uri=CELEX\%3A52014 DC0158 (Zugriff vom 06.07.2016).

124 Siehe Mendelski 2015; Coman 2014. 
demokratische Rechenschaftspflicht der Opposition reduziert wird. Durch ihr inhärentes Evaluierungsdefizit wird die EU zum Unterstützer von partikularistischen, politisierten und instrumentalisierten Justiz- und Rechenschaftsstrukturen, die sich nicht zu langlebigen Institutionen (" perpetually-lived organizations « ${ }^{125}$ ) entwickeln können.

\section{Schlussfolgerung}

Das Evaluierungsdefizit der EU in Hinsicht auf Rechtsstaatlichkeit zeigt drei problematische Aspekte: (1) quantitative Ausrichtung im Praxisvergleich ("Benchmarking «-Ansatz), (2) parteiische und inkonsistente Evaluierung der Rechtsstaatlichkeit, (3) einseitige »rhetorische Aktionen « im Namen der Rechtsstaatlichkeit. Potenzielle Ursachen für das Evaluierungsdefizit der EU sind im politischen und diplomatischen Einfluss sowie der nichtsystematischen jeweiligen Evaluierungsmethodik erkennbar.

Das EU-Evaluierungsdefizit scheint keine einmalige Randerscheinung zu sein, sondern reflektiert ein systematisches, wiederkehrendes Defizit einer inkonsistenten und nichtobjektiven Bewertung der Rechtsstaatlichkeit. Auf diese inadäquate Evaluierungsmethodik wurde bereits von einigen Autoren hingewiesen: erstens bei der Bewertung mittel- und osteuropäischer Länder während der Beitrittsperiode, ${ }^{126}$ zweitens bei der Bewertung der Mitgliedstaaten Rumänien und Bulgarien im Rahmen des CVM-Verfahrens ${ }^{127}$ und drittens bei der Bewertung der Rechtsstaatlichkeit in Ländern der östlichen Partnerschaft ${ }^{128}$ und schließlich, wie hier gezeigt wurde, während der sogenannten "Verfassungskrisen « in Rumänien, Polen und Ungarn. Im Besonderen wurde während dieser Krisen die Rechtsstaatlichkeit nicht systematisch (sowohl zeitlich als auch multidimensional) evaluiert, sondern beschränkte sich auf parteiische Evaluierungsberichte, eine nichtkonstruktive Rhetorik (zum Beispiel "coup d'etat") sowie eine einseitige liberal-konstitutionelle Perspektive mit dem Ziel, politisierte Rechenschaftsinstitutionen (zum Beispiel Verfassungsgerichte) zu schützen. Das Evaluierungsdefizit des Beitrittsprozesses wurde damit in gewisser Weise auf die Mitgliedsstaaten übertragen.

Die sogenannten Rechtsstaatlichkeitskrisen scheinen (im Licht des EU-Evaluierungsdefizits) gar keine reinen Verfassungskrisen zu sein, sondern interinstitutionelle, ideologisch gefärbte Machtkonflikte zwischen politisierten oder niemandem Rechenschaft schuldigen Verfassungsrichtern (Juristokraten), parteiischen Ombudsmännern und äußerst aktiven, ehrgeizigen und politisierenden Regierungspolitikern. Wer genau welche Befugnisse (Gewaltenteilung) und Gesetze überschreitet, ist schwer zu identifizieren und oft nur Auslegungssache. Es wird jedoch ersichtlich, dass trotz dieser gesetzlichen und konzeptionellen Unklarheit (der Verfassung,

125 North et al. 2009.

126 Kochenov 2008.

127 Dimitrov et al. 2014; Toneva-Metodieva 2014.

128 Schimmelfennig 2012; Mendelski 2015; Börzel, Hüllen 2014. 
des Rechtsstaatlichkeitsbegriffs etc.) die EU-Institutionen (Kommission, Parlament) parteiisch in diese innerstaatlichen Machtkonflikte eingreifen, um ihnen nahestehende liberale Umbauagenten zu unterstützen, welche oftmals schon die Legitimität großer Teile der einheimischen Bevölkerung verloren haben.

Es geht nicht darum, herauszufinden, welche der politischen Parteien die Justiz mehr oder weniger politisiert, denn fehlende Rechtsstaatlichkeit ist oft ein systematisches Problem. Wichtig ist, zu erkennen, dass der Respekt für Rechtsstaatlichkeit (und Demokratie) ein Lernprozess ist, welcher objektiv evaluiert werden muss. Diesen Respekt können innerstaatliche Akteure nicht entwickeln, wenn sie (heimlich und indirekt) ermuntert werden, das Recht zu missachten (zum Beispiel durch eine parteiische Evaluierung seitens der EU, transnationaler Netzwerke etc.). Dadurch lernen die sogenannten »illiberalen " Akteure von ihren "liberalen " Gegenspielern, dass die EU ihnen vieles durchgehen lässt, was wiederum dazu verleitet, die Rechtsstaatlichkeit systematisch zu missachten. Der Vorwurf gegenüber der EU wäre also, dass sie sich an die Bedingungen vor Ort anpasst und das politische Spiel der Instrumentalisierung/Politisierung oft nicht nur toleriert, sondern auch selbst mitspielt beziehungsweise verstärkt. Dadurch werden Prozesse der Rechtsstaatlichkeits-und Demokratieförderung sowie die Herausbildung verfassungsrechtlicher »Kulturen des Kompromisses « ${ }^{129}$ trotz kurzfristiger Wahlerfolge »liberaler " Reformbefürworter auf die Dauer untergraben.

Aus einer chronologischen und zeitlich eingebetteten (und damit objektiven) Perspektive erscheinen die jüngsten verfassungspolitischen Machtkrisen als eine populistisch-demokratische Gegenbewegung gegen die zuvor erfolgte, Eliten-getriebene Europäisierung von oben nach unten und im Besonderen gegen die instrumentalisierten und politisierten Justizreformen während der Beitrittsperioden zur EU. Damit könnte man die sogenannten "Verfassungskrisen " in Polen, Ungarn und Rumänien als einen nationalen Schutz gegen Juristokratie und Pathologien der Europäisierung interpretieren. ${ }^{130} \mathrm{Ob}$ sich dieser "patriotische» Widerstand gegen liberale und transnationale Organisationen und Juristokraten-Netzwerke ${ }^{131} \mathrm{zu}$ einem genuin demokratischen Wendepunkt (mit Berücksichtigung des Gemeininteresses) entwickelt oder nur zu einem erneuten, politisierten Zyklus des »ehernen Gesetzes der Oligarchie ", 132 wird die Zukunft zeigen.

Die theoretischen Implikationen dieser Beobachtungen sind aber offensichtlich. Die optimistischen Annahmen vieler Wissenschaftler, in der EU einen neutralen und objektiven Begutachter der Rechtsstaatlichkeit zu sehen, sind kaum noch aufrechtzuerhalten. Genauso muss man die Annahme revidieren, dass horizontale Rechenschaftsinstitutionen (inklusive Verfassungsgerichte und Anti-Korruptionsbehörden) immer nur einen positiven Effekt auf Demokratie und Rechtsstaatlichkeit haben. Die Wirkung dieser horizontalen Rechenschaftsstrukturen hängt vom Kontext ab, 
in den diese Institutionen eingebettet sind. Sie können sowohl positiv sein, wie in den Anfangsperioden der post-kommunistischen Transformation im östlichen Zentraleuropa, ${ }^{133}$ aber auch negativ-pathologisch (wie in Südosteuropa und postsowjetischem Ländern), und somit die Rechtsstaatlichkeit sowohl fördern als auch unterminieren. ${ }^{134}$

Es ist also viel wahrscheinlicher, dass man effektive und unabhängige Rechenschaftsinstitutionen nur über einen "nichtlinearen ", experimentellen Lernprozess ${ }^{135}$ mit nichtintendierten und oft auch pathologischen Folgen zu etablieren vermag. ${ }^{136} \mathrm{Zu}$ einem solchen Prozess gehören natürlich auch das Recht auf Widerstand (gegen das Kapern dieser Institutionen), unzählige Machtkonflikte sowie das Lösen dieser Konflikte mit friedlichen Mitteln. Allerdings wird dieser Lern- und Konfliktlösungsprozess bedingt durch die jeweilige soziale, politische und rechtliche Ordnung, in die die streitenden Akteure eingebettet sind. Externe parteiische Einflussnahme und »rhetorisches Handeln « der EU, die auf bestimmte Erfolgsresultate (zum Beispiel Wahlerfolge der prowestlichen Eliten, Unterzeichnung von Wirtschaftsabkommen) und nicht den kommunikativen Prozess der Verständigung abzielen, können in fragmentierten Politiksystemen die internen Machtkonflikte (trotz kurzfristiger Waffenruhen) verstärken (siehe Rumänien, Polen und Ungarn) und in heterogenen und gespaltenen Gesellschaften zu einer gewalttätigen Ausweitung führen (siehe Ukraine). Zudem riskiert die EU durch ihre einseitige, nichtsystematische Bewertung der Rechtsstaatlichkeit die Transformierung dieses wichtigen Grundrechts in ein politisches und instrumentalisiertes Schlagwort beziehungsweise Instrument gegen Andersdenkende. Rechtsstaatlichkeit als Prinzip der Unparteilichkeit aber kann man nicht durch parteiische Evaluierungen und Rhetorik erreichen. $\mathrm{Da}$ die EU also gezeigt hat, dass sie nicht imstande ist, die Rechtsstaatlichkeit objektiv, konsistent und systematisch zu evaluieren, wäre der Verzicht darauf die angemessenere Verhaltensweise, zumindest solange sie ihre Evaluierungsmethodik nicht ändert.

Wie könnte die Evaluationsmethodik der Rechtstaatlichkeit neu gestaltet werden? Die EU muss sich der Aufgabe stellen, konsistenter und unparteiischer bei der Bewertung der Rechtsstaatlichkeit zu werden. Im Besonderen darf sie nicht über Rechtsstaatlichkeitsverletzungen "liberaler ", EU-freundlicher Eliten hinwegsehen, wenn diese die Justiz oder die Rechenschaftsinstitutionen politisieren, das Recht instrumentalisieren oder Menschenrechte missachten, um ihre Partikularinteressen zu verfolgen. Dies gilt insbesondere für Übergangsperioden ("honeymoon periods ") nach Regimewechseln. Evaluierungsprozesse müssen transparenter gestaltet werden, eine wissenschaftliche Dokumentierung muss obligatorisch sein, und ein ausführlicher Anhang mit allen relevanten einfließenden Informationen sollte integriert werden (zum Beispiel dokumentierte Interviews, Originalfassungen von Experten-

133 Schwartz 2000; Procházka 2002; Ginsburg 2003.

134 Mendelski 2014; Mendelski 2016.

135 Trochev 2008.

136 Mendelski 2015. 
Berichten und Monitoring-Gutachten sowie alternative, kritische Positionen/Kommentare der jeweiligen Regierung und Opposition). Mehr Objektivität und Validität durch Triangulation und Transparenz der Methoden und Informationsquellen kann dieses EU-Problem verringern helfen.

Im Übrigen wären quantitative Auswertungen (inklusive Benchmarking) zur Bewertung der Rechtsstaatlichkeit zu reduzieren und verstärkt qualitative Prozesse in den Blick zu nehmen. Die EU-Konditionalität und Bewertung an spezifische Resultate (zum Beispiel Anzahl der adoptierten Gesetze, besten Praktiken, Anklagen wegen Korruption) zu binden, erweist sich als anfällig für Fehldiagnosen. Stattdessen wäre die Qualität der Prozeduren zu bewerten, die diesen Resultaten unterliegen. Dazu gehören natürlich die Bewertung der Reformen sowie der formalen Legalität (der inneren Moralität des Rechts) durch prozessorientierte Indikatoren. Entsprechendes gilt für die Justizqualität. Deren Bewertung sollte nicht nur (wie im EU Justice Scoreboard) auf Basis von quantitativen Effizienz-Indikatoren erfolgen (zum Beispiel Anzahl der Humanressourcen, Grad der Computerisierung, Länge der Gerichtsverfahren etc.), sondern viel stärker die prozedurale Prozessqualität berücksichtigen, also etwa die unparteiische und rechenschaftspflichtige Auswahl geeigneter Richter und Staatsanwälte oder den Grad der Koordinierung und der »loyalen Kooperation « der Akteure in der Justiz und Politik.

Die EU hat sich auf eine reaktive, problemorientierte und punktuelle Sichtweise auf Rechtsstaatlichkeit eingespielt. Stattdessen müsste sie eine holistische, systematische und multiplikative Methodik zur Bewertung der Rechtsstaatlichkeit einführen, welche die interdependenten Beziehungen zwischen einzelnen Rechtsstaatlichkeit-Dimensionen (beispielsweise zwischen Justizunabhängigkeit und Rechenschaftspflicht) sowie die soziale, historische und politische Einbettung der Rechtsstaatlichkeit berücksichtigt. Nur dadurch könnten diverse Pathologien der Europäisierung reduziert werden.

\section{Literatur}

Apeldoorn, Bastian van 2003. Transnational capitalism and the struggle over European integration. London: Routledge.

Avery, Graham 2015. "Enlargement policy in perspective ", in EU member states and enlargement towards the Balkans. EPC Issue Paper No. 79, hrsg. v. Balfour, Rosa; Stratulat, Corina, S. 11-18. Brüssel: European Policy Centre.

Belloni, Roberto; Strazzari, Francesco 2014. »Corruption in post-conflict Bosnia-Herzegovina and Kosovo: a deal among friends ", in Third World Quarterly 35, 5, S. 855-871.

Beširević, Violeta 2014. "Governing without judges: the politics of the Constitutional Court in Serbia ", in International Journal of Constitutional Law 12, 4, S.954-979.

Blauberger, Michael 2016. "Europäischer Schutz gegen nationale Demokratiedefizite? ", in Leviathan 44, 2, S. 280-302.

Blokker, Paul 2016. "EU democratic oversight and domestic deviation from the rule of law: sociological reflections", in Reinforcing the rule of law oversight in the European Union, hrsg. v. Closa, Carlos; Kochenov, Dimitry, Kapitel 12. Cambridge: Cambridge University Press.

Bobek, Michal; Kosar, David 2014. "Global solutions, local damages: a critical study in judicial councils in Central and Eastern Europe ", in German Law Journal 15, 2, S. 1257-1292.

Bogdandy, Armin von; Sonnevend, Pál. Hrsg. 2015. Constitutional crisis in the European constitutional area: theory, law and politics in Hungary and Romania. Oxford: Hart. 
Börzel, Tanja A. 2015. "The noble west and the dirty rest? Western democracy promoters and illiberal regional powers ", in Democratization 22, 3, S. 519-535.

Börzel, Tanja A. 2016. »Building member states: how the EU promotes political change in its new members, accession candidates, and Eastern neighbors ", in Geopolitics, History \& International Relations 8, 1, S. 76-112.

Börzel, Tanja A.; Pamuk, Yasemin 2012. "Pathologies of Europeanisation: fighting corruption in the Southern Caucasus «, in West European Politics 35, 1, S. 79-97.

Börzel, Tanja A.; Hüllen, Vera van 2014. "One voice, one message, but conflicting goals: cohesiveness and consistency in the European neighbourhood policy ", in Journal of European Public Policy 21, 7, S. 1033-1049.

Camp, Robert C. 1995. Business process benchmarking: finding and implementing best practices. Milwaukee: ASQC Quality Press

Caplan, Richard 2005. "Who guards the guardians? International accountability in Bosnia «, in International Peacekeeping 12, 3, S. 463-476.

Capussela, Andrea L. 2015. State-building in Kosovo: democracy, corruption and the EU in the Balkans. London: IB Tauris.

Carothers, Thomas 2003. Promoting the rule of law abroad: the problem of knowledge. Rule of Law Series 34. Washington: Carnegie Endowment for International Peace.

Carrera, Sergio; Guild, Elspeth; Hernanz, Nicholas 2013. The triangular relationship between fundamental rights, democracy and the rule of law in the EU: towards an EU Copenhagen mechanism. Brüssel: Centre for European Policy Studies.

Channell, Wade 2006. "Lessons not learned: problems with Western aid for law reform in postcommunist countries ", in Journal of Comparative Law 1, 2, S. 321-337.

Closa, Carlos; Kochenov, Dimitry. Hrsg. 2016. Reinforcing rule of law oversight in the European Union. Cambridge: Cambridge University Press.

Coman, Ramona 2014. "Quo vadis judicial reforms? The quest for judicial independence in Central and Eastern Europe «, in Europe-Asia Studies 66, 6, S. 892-924.

Dimitrov, Georgi; Haralampiev, Kaloyan; Stoychev, Stoycho; Toneva-Metodieva, Iinka 2014. The cooperation and verification mechanism: shared political irresponsibility between the European Commission and the Bulgarian governments. Sofia: St. Kliment Ohridski University Press.

Di Puppo, Lili 2010. "Anti-corruption interventions in Georgia ", in Global Crime 11, 2, S. $220-236$.

Elbasani, Arolda. Hrsg. 2013. European integration and transformation in the western Balkans. Europeanization or business as usual? New York: Routledge.

Elias, Norbert 1992. Studien über die Deutschen. Machtkämpfe und Habitusentwicklung im 19. und 20. Jabrbundert. Frankfurt a. M.: Suhrkamp.

Europäische Kommission 2006. Romania. May 2006 Monitoring Report, SEC (2006) 596, 16.05. 2006. Brüssel.

Europäische Kommission 2008. Supporting document accompanying the report from The Commission to the European Parliament and the Council on Progress in Romania under the cooperation and verification mechanism. Romania: Technical Update. COM(2008) 494 final, 23.07.2008. Brüssel.

Europäische Kommission 2012. Report from the Commission to the European Parliament and the Council on progress in Romania under the co-operation and verification mechanism. COM(2012) 410 final, 18.07.2012. Brüssel.

Europäische Kommission 2014. Communication: a new EU framework to strengthen the rule of law. COM(2014) 158 Final. Brüssel.

Fuller, Lon L. 1969. The morality of law. New Haven, London: Yale University Press.

Garoupa, Nuno; Ginsburg, Tom 2009. "The comparative law and economics of judicial councils", in Berkeley Journal of International Law 27, 1, S. 53-83.

Ghiciusca, Andrei 2007. "Basescu incalca independenta Justitiei “, in Ziua vom 10. Juli 2007.

Ginsburg, Tom 2003. Judicial review in new democracies: constitutional courts in Asian cases. New York: Cambridge University Press

Goetz, Klaus H.; Zubek, Radowlaw 2007. "Government, parliament and law-making in Poland ", in The Journal of Legislative Studies 13, 4, S. 517-538.

Greiffenhagen, Martin 1999. Kulturen des Kompromisses. Opladen: Leske + Budrich. 
Haas, Peter M. 1992. "Introduction: epistemic communities and international policy coordination ", in International Organization 46, 1, S. 1-35.

Habermas, Jürgen 1992. Faktizität und Geltung: Beiträge zur Diskurstheorie des Rechts und des demokratischen Rechtsstaates. Frankfurt a. M.: Suhrkamp.

Hayden, Robert M. 2005. »Democracye without a demos? The Bosnian constitutional experiment and the intentional construction of nonfunctioning states ", in East European Politics o Societies 19, 2, S. 226-259.

Hirschl, Ran 2009. Towards juristocracy: the origins and consequences of the new constitutionalism. Cambridge, London: Harvard University Press.

Ivanova, Ivanka 2013. "Rule of law in Bulgaria and the influence of EU accession ", in The triangular relationship between fundamental rights, democracy and the rule of law in the EU: towards an EU Copenhagen mechanism, hrsg. v. Carrera, Sergio; Guild, Elspeth; Hernanz, Nicholas, S. 98-106. Brüssel: Center for European Policy Studies.

Jakab, András; Kochenov, Dimitry. Hrsg. 2016. The enforcement of EU law and values. Oxford: Oxford University Press.

Kantorowicz, Jaroslaw; Garoupa, Nuno 2016. "An empirical analysis of constitutional review voting in the polish constitutional tribunal, 2003-2014 «, in Constitutional Political Economy 27,1, S. 66-92.

Kochenov, Dimitry 2008. EU enlargement and the failure of conditionality. Pre-accession conditionality in the fields of democracy and the rule of law. Alphen aan den Rijn: Wolters Kluwer.

Kilpatrick, Claire 2015. „On the rulc of law and economic emergency: the degradation of basic legal values in Europe's bailouts ", in Oxford Journal of Legal Studies 35, 2, S. 325-353.

Kostanyan, Hrant 2016. Why Moldova's European integration is failing. CEPS Commentary vom 3. März 2016. Brüssel: Center for European Policy Studies.

Kreuder-Sonnen, Christian 2016. "Beyond integration theory: the (anti-) constitutional dimension of European crisis governance", in Journal of Common Market Studies (Online-Version). DOI: $10.1111 / \mathrm{jcms} .12379$.

Kuzmova, Yoana 2014. "Bulgarian specialized criminal court after one year: a misplaced transplant, an instrument of justice, or a tool of executive power? «, in Boston University International Law Journal 32, S. 227-262.

Maatsch, Aleksandra 2015. Empowered or disempowered? The role of national parliaments during the reform of European economic governance. MPIfG Discussion Paper 15/10. Köln: Max-Planck-Institut für Gesellschaftsforschung.

Magen, Amichai; Morlino, Leonardo 2009. International actors, democratization and the rule of law. Anchoring democracy? New York: Routledge.

Maravall, José Maria 2003. "The rule of law as a political weapon «, in Democracy and the Rule of Law 5, S. 261-301.

Matthijs, Matthias 2014. "Mediterranean blues: the crisis in Southern Europe", in Journal of Democracy 25, 1, S. 101-115.

Mendelski, Martin 2011. "Rulc of law reforms in the shadow of clientelism: the limits of the EU's transformative power in Romania ", in Polish Sociological Review 2, 174, S. 235-253.

Mendelski, Martin 2012. "EU-driven judicial reforms in Romania: a success story? ", in East European Politics 28, 1, S. 23-42.

Mendelski, Martin 2014. The limits of the European Union's transformative power: patbologies of Europeanization and rule of law reform in Central and Eastern Europe. Doctoral thesis, University of Luxembourg.

Mendelski, Martin 2015. "The EU's pathological power: the failure of external rule of law promotion in South Eastern Europe", in Southeastern Europe 39, 3, S. 318-346.

Mendelski, Martin 2016. "Europeanization and the rule of law: Towards a pathological turn «, in Southeastern Europe (im Erscheinen).

Michels, Robert 1915. Political parties: a sociological study of the oligarchical tendencies of modern democracy. New York: Hearst's International Library Company.

Morlino, Leonardo; Sadurski, Wojciech. Hrsg. 2010. Democratization and the European Union: comparing central and eastern European post-communist countries. New York: Routledge.

Müller, Jan-Werner 2015. "Should the EU protect democracy and the rule of law inside member states? ", in European Law Journal 21, 2, S. 141-160. 
Mungiu-Pippidi, Alina 2014. "The transformative power of Europe revisited", in Journal of Democracy 25, 1, S. 20-32.

Natorski, Michal 2013. "Reforms in the judiciary of Ukraine: domestic practices and the EU's policy instruments", in East European Politics 29, 3, S. 358-375.

North, Douglas C.; Wallis, John J.; Weingast, Barry R. 2009. Violence and social orders. A conceptual framework for interpreting recorded buman history. Cambridge: Cambridge University Press.

Parau, Cristina E. 2015. "Explaining governance of the judiciary in Central and Eastern Europe: external incentives, transnational elites and parliamentary inaction *, in Europe-Asia Studies 67,3, S. $409-442$.

Pech, Laurent 2016. »The EU as a global rule of law promoter: the consistency and effectiveness challenges ", in Asia Europe Journal 14, 1, S. 7-24.

Piana, Daniela; Dallara, Cristina 2015. Networking the rule of law: how change agents reshape judicial governance in the EU. Farnham: Ashgate Publishing.

Polanyi, Karl 1944. The great transformation. The political and economic origins of our time. Boston: Beacon Press.

Popoya, Maria 2012. Politicized justice in emerging democracies: a study of courts in Russia and Ukraine. Cambridge: Cambridge University Press.

Procházka, Radoslav 2002. Mission accomplished: on founding constitutional adjudication in Central Europe. Budapest: Central Europcan Universiry Press.

Przeworski, Adam 1991. Democracy and the market: political and economic reforms in Eastern Europe and Latin America. Cambridge: Cambridge University Press.

Radwan, Arkadiusz 2016. Chess-boxing around the rule of law - Polish constitutionalism at trial. Allerhand Working Paper 13/2016. http://papers.ssrn.com/sol3/papers.cfm?abstract_id=27 48746 (Zugriff vom 07.06.2016).

Risse, Thomas; Sikkink, Kathryn 1999. "The socialization of international human rights norms into domestic practices", in The power of human rights: intemational norms and domestic change, hrsg. v. Ropp, Stephen C.; Sikkink, Kathryn, S. 1-38. Cambridge: Cambridge University Press.

Risteska, Marija 2013. "The role of the EU in promoting good governance in Macedonia: towards efficiency and effectiveness or deliberative democracy? ", in Nationalities Papers 41, 3, S. 431-446.

Robinson, William I. 2004. A theory of global capitalism. Baltimore, London: The Johns Hopkins University Press.

Sadurski, Wojciech 2004. "Accession's democracy dividend: the impact of the EU enlargement upon democracy in the new member states of Central and Eastern Europe«, in European Law Journal 10, 4, S. 371-401.

Schedler, Andreas 1999. The self-restraining state: power and accountability in new democracies. Boulder: Lynne Rienner Publishers.

Schimmelfennig, Frank 1997. "Rhetorisches Handein in der internationalen Politik ", in Zeitschrift für internationale Beziebungen 4, 2, S.219-254.

Schimmelfennig, Frank 2001. "The community trap: liberal norms, rhetorical action, and the Eastern enlargement of the European Union «, in International Organization 55, 1, S. 47-80.

Schimmelfennig, Frank 2012. "Europeanization beyond Europe", in Living Reviews in European Governance 7, 1, S. 1-31.

Schimmelfennig, Frank; Sedelmeier, Ulrich 2005. The Europeanization of Central and Eastern Europe. Ithaca: Cornell University Press.

Schmitter, Philippe C. 2004. "The ambiguous virtues of accountability «, in Journal of Democracy 15,4, S. 47-60.

Schwartz, Herman 2000. The struggle for constitutional justice in post-communist Europe. Chicago: University of Chicago Press.

Shapiro, Martin M. 1988. Who guards the guardians? Judicial control of administration. Athens: University of Georgia Press.

Shklar, Judith N. 1987. "Political theory and the rule of law ", in The rule of law: ideal or ideology, hrsg. v. Hutchinson, Alan C.; Monahan, Patrick, S. 1-16. Toronto: Carswell.

Slapin, Jonathan B. 2015. "How European Union membership can undermine the rule of law in emerging democracies «, in West European Politics 38, 3, S. 627-648. 
Streeck, Wolfgang 2015. "Heller, Schmitt and the Euro", in European Law Journal 21, 3, S. $361-370$.

Tamanaha, Brian Z. 2004. On the rule of law: history, politics, theory. Cambridge: Cambridge University Press.

Teodorescu, Bogdan; Sultanescu, Dan 2006. Revolutie portocalie in Romania. Bukarest: Fundatia PRO.

Toneva-Metodieva, Linka 2014. "Beyond the carrots and sticks paradigm: rethinking the cooperation and verification mechanism experience of Bulgaria and Romania ", in Perspectives on European Politics and Society 15, 4, S. 534-551.

Trochev, Alexei 2008. Judging Russia: the role of the constitutional court in Russian politics 1990-2006. Cambridge: Cambridge University Press.

Tudoroiu, Theodor 2015. "Democracy and state capture in Moldova ", in Democratization 22, 4, S. 655-678.

Venice Commission 2016. Opinion on Amendments to the Act of 25 June 2015 on the Constitutional Tribunal of Poland. Adopted by the Venice Commission at its 106 th Plenary Session, Opinion no. 833/2015, Venedig, 11. März 2016.

White, Jonathan 2015. "Emergency Europe», in Political Studies 63, 2, S. 300-318. 
Zusammenfassung: Liberale Repräsentanten der Europäischen Union (EU) sowie Anhänger eines liberalen Konstitutionalismus beurteilen die jüngsten »Verfassungskrisen « in Zentralund Osteuropa als einen Angriff auf Demokratie und Rechtsstaatlichkeit. Diese einseitige Bewertung resultiert unter anderem aus einer inadäquaten Methodologie zur Evaluierung der Rechtsstaatlichkeit. Der Autor dieses Beitrags identifiziert ein Defizit der EU bei der Evaluierung der Rechtsstaatlichkeit, welches sich in drei Kernproblematiken wiederspiegelt: (1) quantitativer "Je mehr desto besser «-Ansatz, (2) parteiische Evaluierung der Rechtsstaatlichkeit, (3) parteiliches »rhetorisches Handeln « im Namen der Rechtsstaatlichkeit. Das Evaluierungsdefizit der EU wird anhand ausgewählter Beispiele zu Justizreformen und "Verfassungskrisen « in Zentral- und Osteuropa (beispielsweise Rumänien, Polen, Ungarn) dargestellt. Es wird argumentiert, dass die EU aufgrund ihres Evaluierungsdefizits nicht imstande ist, die Rechtsstaatlichkeit objektiv und systematisch zu beurteilen. Die EU sollte deshalb die Rechtsstaatlichkeit in ihren Mitglieds- und Beitrittsländern nicht mehr evaluieren, es sei denn, sie ändert ihre defizitäre Evaluationsmethodik, indem sie zum Beispiel den Politikempfehlungen dieses Beitrags folgt.

Stichworte: Rechtsstaatlichkeit, Europäische Union, Evaluierungsdefizit, Verfassungskrisen, Rumänien, Polen, Ungarn, Europäisierung

\section{The European rule of law evaluation deficit}

Summary: Liberal representatives of the European Union (EU) and adherents of liberal constitutionalism assess the recent "constitutional crises" in Central and Eastern Europe as an attack on democracy and the rule of law. This one-sided assessment results, among others, from an inadequate rule of law evaluation methodology. The author of this paper identifies the EU's rule of law evaluation deficit, which is reflected in three fundamental problems: (1) A quantitative "more is better" mindset, (2) A biased assessment of the rule of law, (3) Partisan "rhetoric action « in the name of the rule of law. The EU's evaluation deficit is illustrated with selected examples of judicial reform and constitutional crises in CEE (e.g. Romania, Poland, Hungary). It is argued that given the EU's inability to objectively and systematically assess the rule of law, the EU should either abstain from evaluating rule of law in member states and candidate countries or change its deficient evaluation approach, for instance by following the policy advice provided in this paper.

Keywords: rule of law, European Union, evaluation deficit, constitutional crises, Romania, Poland, Hungary, Europeanization

\section{Autor}

Martin Mendelski

Max-Planck-Institut für Gesellschaftsforschung

Paulstraße 3

50676 Köln

mendelski@mpifg.de 